https://helda.helsinki.fi

\title{
Survey of local algorithms
}

\section{Suomela, Jukka}

\section{3}

Suomela , J 2013 , ' Survey of local algorithms ' , ACM Computing Surveys , vol. 45 , no. 2 , pp. Article No. 24 . https://doi.org/10.1145/2431211.2431223

http://hdl.handle.net/10138/40800

https://doi.org/10.1145/2431211.2431223

submittedVersion

Downloaded from Helda, University of Helsinki institutional repository.

This is an electronic reprint of the original article.

This reprint may differ from the original in pagination and typographic detail.

Please cite the original version. 


\title{
SURVEY OF LOCAL ALGORITHMS
}

\author{
JUKKA SUOMELA
}

\begin{abstract}
A local algorithm is a distributed algorithm that runs in constant time, independently of the size of the network. Being highly scalable and fault-tolerant, such algorithms are ideal in the operation of large-scale distributed systems. Furthermore, even though the model of local algorithms is very limited, in recent years we have seen many positive results for non-trivial problems. This work surveys the state-of-the-art in the field, covering impossibility results, deterministic local algorithms, randomised local algorithms, and local algorithms for geometric graphs.

Categories And SUBJeCt DesCriptors: C.2.4 [Computer-Communication Networks]: Distributed Systems; F.1.1 [Computation by Abstract Devices]: Models of Computation; F.2.2 [Analysis of Algorithms and Problem Complexity]: Nonnumerical Algorithms and Problems - computations on discrete structures.
\end{abstract}

General Terms: Algorithms, Theory.

ADDITIONAL KEY WORDS: Local algorithms.

\section{INTRODUCTION}

The running time of an algorithm typically increases with the size of the input - sorting one thousand names takes longer than sorting one hundred names. Only the most trivial problems can be solved in constant time, independently of the size of the input. There is one notable exception, though: there are non-trivial distributed algorithms that run in constant time.

In a distributed algorithm, the same computer network is both the input and the system that solves the problem; hence a larger input also implies a larger number of parallel computers. This does not make problems trivial to solve fast, but it turns out that there are several examples of computational problems that can be solved by using a constant-time distributed algorithm. Such algorithms are known as local algorithms.

1.1. Local algorithms. A local algorithm is a distributed algorithm that runs in a constant number of synchronous communication rounds, independently of the number of nodes in the network. Put otherwise, the output of a node in a local algorithm is a function of the input available within a constant-radius neighbourhood of the node.

Research on local algorithms was pioneered by Angluin [5], Linial [109], and Naor and Stockmeyer [121]. Angluin [5] studied the limitations of

Date: 6 th November 2011 - final version.

(C) ACM, 2011. This is the author's version of the work. It is posted here by permission of ACM for your personal use. Not for redistribution. The definitive version will be published in ACM Computing Surveys. 
anonymous networks without any unique identifiers. Linial [109] proved seminal negative results for the case where each node has a unique identifier. Naor and Stockmeyer [121] presented the first nontrivial positive results.

This work is a survey of local algorithms. We focus on algorithms whose running time and performance guarantees are independent of the number of nodes in the network - put simply, these are algorithms that could be used to control infinitely large networks in finite time. For a more general discussion on distributed algorithms, see, e.g., Peleg [127] and Elkin [38].

Many of the negative results cited in this survey were not originally stated as negative results for local algorithms; they are more general results which, as a corollary, imply that a particular problem cannot be solved by any local algorithm. The emphasis is on results that have nontrivial implications for local algorithms. Further impossibility results for distributed algorithms are presented in the surveys by Lynch [114] and Fich and Ruppert [41].

1.2. Structure of this work. We begin with some essential definitions in Section 2. Section 3 reviews the advantages and applications of local algorithms. Section 4 introduces the computational problems that we use as examples throughout this work, and Section 5 discusses what information each node has available in a local algorithm. Section 6 reviews negative results: what cannot be computed with a local algorithm. Section 7 reviews positive results: which deterministic local algorithms are known. In Section 8 we study the power of randomness, in comparison with deterministic local algorithms. Section 9 studies local algorithms in a geometric setting, in which each node knows its coordinates. Section 10 concludes this survey with some open problems.

For a quick summary of the negative results for deterministic local algorithms, see Tables 1 and 2 on page 18. The positive results for deterministic local algorithms are summarised in Tables 3 and 4 on pages 23-24. Many of the results summarised in the tables are corollaries that have not been stated explicitly in the literature.

\section{Definitions}

In this survey, all graphs are simple and undirected unless otherwise mentioned. Terminology related to directed graphs is introduced in Section 5.4, and geometric graphs such as unit-disk graphs are defined in Section 9.1.

2.1. Graphs. For a graph $\mathcal{G}=(V, E)$, we use the following notation and terminology. An undirected edge between nodes $u \in V$ and $v \in V$ is represented by an unordered pair $\{u, v\} \in E$. We write $\operatorname{deg}(v)$ for the degree (number of neighbours) of node $v \in V$. A node $v \in V$ is isolated if $\operatorname{deg}(v)=0$.

Graph $\mathcal{G}$ is $k$-regular if $\operatorname{deg}(v)=k$ for each $v \in V$. Graph $\mathcal{G}$ is bipartite if $V=V_{1} \cup V_{2}$ for disjoint sets $V_{1}$ and $V_{2}$ such that each edge $e \in E$ is of the form $e=\{u, v\}$ for $u \in V_{1}$ and $v \in V_{2}$. The complete graph on $n$ nodes is denoted by $K_{n}$.

2.2. Neighbourhoods. We use $d_{\mathcal{G}}(u, v)$ to denote the shortest-path distance (number of edges, hop count) between nodes $u$ and $v$ in graph $\mathcal{G}$, and

$$
B_{\mathcal{G}}(v, r)=\left\{u \in V: d_{\mathcal{G}}(u, v) \leq r\right\}
$$




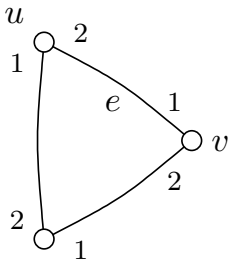

(a)

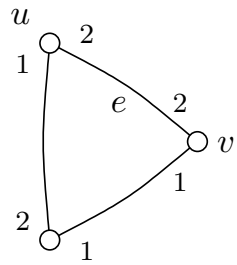

(b)

FiguRE 1. A communication graph with a port numbering.

to denote the radius- $r$ neighbourhood of node $v$ in graph $\mathcal{G}$. We write $\mathcal{G}(v, r)$ for the subgraph of $\mathcal{G}$ induced by $B_{\mathcal{G}}(v, r)$. We occasionally refer to the subgraph $\mathcal{G}[v, r]$ of $\mathcal{G}(v, r)$; graph $\mathcal{G}[v, r]$ is constructed from $\mathcal{G}(v, r)$ by removing the edges $\{s, t\}$ with $d_{\mathcal{G}}(v, s)=d_{\mathcal{G}}(v, t)=r$.

2.3. Communication graph. Throughout this work, graph $\mathcal{G}=(V, E)$ is the communication graph of a distributed system: each node $v \in V$ is a computational entity and an edge $\{u, v\} \in E$ denotes that nodes $u$ and $v$ can communicate with each other.

Often we have to make assumptions on the structure of the communication graph. Among others, we study the family of bounded-degree graphs. In this case we assume that there is a known constant $\Delta$, and any node in any communication graph $\mathcal{G}$ that we may encounter is guaranteed to have at most $\Delta$ neighbours.

2.4. Port numbering. We assume that there is a port numbering (local edge labelling) $[5,11,150]$ available for the communication graph $\mathcal{G}$. This means that each node of $\mathcal{G}$ imposes an ordering on its incident edges. Thus each edge $\{u, v\} \in E$ has two natural numbers associated with it: the port number at node $u$, denoted by $p(u, v)$, and the port number at node $v$, denoted by $p(v, u)$. If $p(u, v)=i$, we also say that the neighbour $i$ of $u$ is $v$.

See Figure 1 for an illustration. Figure 1a shows one possible way to assign the port numbers in a 3 -cycle. The port number at node $u$ for edge $e=\{u, v\} \in E$ is $p(u, v)=2$, and the port number at node $v$ for edge $e$ is $p(v, u)=1$. The neighbour 2 of $u$ is $v$. Figure $1 \mathrm{~b}$ shows another way to assign the port numbers in the same graph.

2.5. Model of distributed computing. We use Linial's [109] model of computation; Peleg [127] calls it the local model. Each node in the system executes the same algorithm $\mathcal{A}$. Initially, each node $v \in V$ knows a taskspecific local input $i_{v}$. Each node $v \in V$ has to produce a local output $o_{v}$. We always assume that $\operatorname{deg}(v)$ is part of the local input $i_{v}$. The local input $i_{v}$ may also contain auxiliary information such as unique node identifiers; this is discussed in more detail in Section 5.

The distributed system operates in a synchronous manner. Let $r$ be the number of synchronous communication rounds. In each round $i=1,2, \ldots, r$, the following operations are performed, in this order:

(1) Each node performs local computation.

(2) Each node $v$ sends one message to each port $1,2, \ldots, \operatorname{deg}(v)$. 
(3) Each node $v$ receives one message from each port $1,2, \ldots, \operatorname{deg}(v)$. Finally, after round $r$, each node $v \in V$ performs local computation and announces its local output $o_{v}$. The size of a message is unbounded and local computation is free.

Example 1. Consider the graph in Figure 1a. If node $u$ sends a message $m$ to port 2 in round $i$, the same message $m$ is received by node $v$ from port 1 in the same round $i$. Note that node $u$ can include the outgoing port number 2 in message $m$; then the receiver $v$ learns that the edge number 1 at $v$ equals the edge number 2 at its neighbour $u$.

2.6. Local algorithm and local horizon. We say that $\mathcal{A}$ is a local algorithm if the number of communication rounds $r$ is a constant. The constant $r$ may depend on the parameters of the problem family; for example, if we study bounded-degree graphs, the value of $r$ may depend on the parameter $\Delta$. However, the value of $r$ cannot depend on the problem instance; in particular, it does not depend on the number of nodes in graph $\mathcal{G}$.

The constant $r$ is called the local horizon of the local algorithm. In $r$ synchronous communication rounds, information can be propagated for at most $r$ hops in the network. The output $o_{v}$ of a node $v \in V$ may depend on the local inputs $i_{u}$ for all $u \in B_{\mathcal{G}}(v, r)$; however, it cannot depend on the local input $i_{u}$ for any $u \notin B_{\mathcal{G}}(v, r)$. A decision must be made based on the information that is available within the local horizon.

Throughout this work, the original definition of a local algorithm by Naor and Stockmeyer [121] is used: $r$ must be a constant. In many papers, the term "local algorithm" is used in a less strict manner, and terminology such as "strictly local algorithm" or " $O(1)$-local algorithm" is used to refer to the case of a constant $r$.

2.7. Local approximation. An $\alpha$-approximation algorithm is an algorithm that produces a feasible output, and the utility of the output is guaranteed to be within factor $\alpha$ of the utility of an optimal solution. We use the convention that $\alpha \geq 1$ for both minimisation and maximisation problems [12]. Hence, for a minimisation problem, an $\alpha$-approximation algorithm produces a feasible solution with a cost of at most $\alpha$. OPT where OPT is the cost of an optimal solution, and for a maximisation problem, an $\alpha$-approximation algorithm produces a feasible solution with a utility of at least $\mathrm{OPT} / \alpha$ where OPT is the utility of an optimal solution.

A local $\alpha$-approximation algorithm is an $\alpha$-approximation algorithm and a local algorithm. A local approximation scheme is a family of local algorithms such that for each $\varepsilon>0$ there is a local $(1+\varepsilon)$-approximation algorithm.

2.8. Distributed constant-size problem. We say that a problem is of distributed constant size if $\mathcal{G}$ is a bounded-degree graph and the size of the local input $i_{v}$ is bounded by a constant. If we have a local algorithm for a distributed constant-size problem, then each node needs to transmit and process only a constant number of bits; therefore local computations can be done in constant time, and the size of the local output $o_{v}$ is bounded by a constant as well. Informally, a local algorithm for a distributed constant-size problem runs in constant time, regardless of the details of the model of 
distributed computing; we do not need to exploit the unbounded size of messages and unlimited local computation.

\section{Advantages And applications}

The problems that we study in this survey are typically inspired by applications related to the operation of communication networks: for example, monitoring communication networks, scheduling the activities of the nodes in a network, or routing data in a network. We will discuss the problems in more detail in Section 4, but let us first study why it is advantageous to use local algorithms in such applications. In addition to discussing the practical uses of local algorithms in communication networks, we will also explore connections between local algorithms and other fields of computer science.

3.1. Fault tolerance and robustness. A local algorithm is not only highly scalable but also fault-tolerant. A local algorithm recovers efficiently from failures, changes in the network topology, and changes in the input [121]. If the input of a node $v \in V$ changes, this only affects the output within $B_{\mathcal{G}}(v, r)$.

In particular, a local algorithm can be used to maintain a feasible solution in a dynamic graph in which edges and nodes are added and deleted [39]. In the case of distributed constant-size problems, a local algorithm supports arbitrary updates in the graph in constant time per operation.

Naor and Stockmeyer [121] point out the connection between local algorithms and self-stabilising algorithms [35, 36, 131]. A self-stabilising algorithm arrives at a legitimate state - "stabilises" - in finite time regardless of the initial states of the nodes. Work on self-stabilising algorithms $[14,15]$ provides, as a simple special case, a mechanical way to transform a constant-time deterministic distributed algorithm into a self-stabilising algorithm that stabilises in constant time; see Lenzen et al. [105] for more details on the connection between local and self-stabilising algorithms.

3.2. Value of information. In the model of local algorithms, we assume that local computation is free. Hence our focus is primarily on the amount of information needed in distributed decision making: what can we do with the information that is available in the constant-radius neighbourhood of a node. Positive and negative results for local algorithms can be interpreted as information-theoretic upper and lower bounds; they give insight into the value of information $[124,125]$.

3.3. Other models of computing. Local algorithms are closely connected to circuit complexity and the complexity class $\mathrm{NC}^{0}$ [1]: if a distributed constant-size problem can be solved with a local algorithm, then for any bounded-degree graph $\mathcal{G}$ there is a bounded-fan-in Boolean circuit that maps the local inputs to the local outputs, and the depth of the circuit is independent of the size of $\mathcal{G}$. As pointed out by Wattenhofer and Wattenhofer [141], a local algorithm provides an efficient algorithm in the PRAM model, but a PRAM algorithm is not necessarily local.

Sterling [133] shows that lower bounds for local algorithms can be applied to derive lower bounds in the tile-assembly model. Gibbons [57] points out 
that the envisioned shape-shifting networks will require not only advances in hardware, but also novel local algorithms.

3.4. Sublinear-time centralised algorithms. A local algorithm for a distributed constant-size problem provides a linear-time centralised algorithm: simply simulate the local algorithm for each node. Parnas and Ron [126] show that in some cases it is possible to use a local algorithm to design a sublinear-time (or even constant-time) centralised approximation algorithm; see also Nguyen and Onak [122] and Floréen et al. [48].

For example, consider a local approximation algorithm $\mathcal{A}$ for the vertex cover problem (see Section 4.4 below for the definition). For a given input graph $\mathcal{G}$, algorithm $\mathcal{A}$ produces a feasible and approximately optimal vertex cover $C$. From the point of view of a centralised algorithm, the local algorithm $\mathcal{A}$ can be interpreted as an oracle with which we can access the cover $C$ : for any given node $v$, we can efficiently determine whether $v \in C$ or not by simulating algorithm $\mathcal{A}$ at node $v$. Therefore we can estimate the size of the cover $C$ by sampling nodes uniformly at random; for each node we determine whether it is in $C$ or not. Furthermore, as we know that $C$ is approximately optimal, estimating the size of $C$ allows us to estimate the size of the minimum vertex cover of graph $\mathcal{G}$ as well.

These kinds of algorithms can be used to obtain information about the global properties of very large graphs. Lovász [110] gives examples of such graphs: the Internet, the social network of all living people, the human brain, and crystal structures. Many of these are not explicitly given and not completely known; however, it may be possible to obtain information about these graphs by sampling nodes and their local neighbourhoods. From this perspective, the sublinear-time algorithm by Parnas and Ron [126] puts together neighbourhood sampling and a local approximation algorithm to estimate the global properties of huge graphs.

\section{Problems}

Now we proceed to give the definitions of the computational problems that we discuss in this survey. Most of these problems are classical combinatorial problems; for more details and background, see textbooks on graph theory [34], combinatorial optimisation [88, 123], NP-completeness [55], and approximation algorithms $[12,139]$.

4.1. Encoding of input and output. When we study local algorithms, we assume that the problem instance is given in a distributed manner: each node in the communication graph $\mathcal{G}$ knows part of the input. For graph problems, the connection between the communication graph $\mathcal{G}$ and the structure of the problem instance is usually straightforward: we simply assume that the communication graph $\mathcal{G}$ is our input graph - note that we can easily modify the communication graph by removing edges and nodes that are irrelevant from the perspective of the graph problem that we are solving. For more general packing and covering problems (such as the set cover problem or packing LPs) there is more freedom. However, it is fairly natural to represent such problems in terms of bipartite graphs, and this has been commonly used in the literature $[18,98,125]$. We follow this convention. 
The exact definitions of the local input $i_{v}$ and output $o_{v}$ are usually fairly straightforward. For unweighted graph problems, we do not need any task-specific information in the local input $i_{v}$; the structure of the communication graph $\mathcal{G}$ is enough. For weighted graph problems, the local input $i_{v}$ contains the weight of node $v$ and the weights of the incident edges. Hence all unweighted graph problems in bounded-degree graphs are distributed constant-size problems; weighted problems are distributed constant-size problems if the weights are represented with a bounded number of bits.

If the output is a subset $X \subseteq V$ of nodes, then the local output $o_{v}$ is simply one bit of information: whether $v \in X$ or not. If the output is a subset $X \subseteq E$ of edges, then the local output $o_{v}$ contains one bit for each incident edge $e$. The algorithm must produce a correct output no matter how we choose the port numbers in the communication graph $\mathcal{G}$.

4.2. Independent sets. A set of nodes $I \subseteq V$ is an independent set if no two nodes in $I$ are adjacent, that is, there is no edge $\{u, v\} \in E$ with $u \in I$ and $v \in I$.

In typical applications, graph $\mathcal{G}$ is interpreted as a conflict graph: an edge $\{u, v\} \in E$ indicates that the activities of $u$ and $v$ conflict with each other. For example, if $\mathcal{G}$ is a wireless network, an edge $\{u, v\}$ may indicate that the radio transmission of device $u$ interferes with device $v$ [77]. In such a setting, an independent set $I$ is a conflict-free set of activities: all devices in $I$ can be active simultaneously. Usually we are interested in finding large independent sets.

An independent set $I$ is maximal if it cannot be extended, that is, $I \cup\{v\}$ is not an independent set for any $v \in V \backslash I$. In a centralised setting, a maximal independent set is easy to find by using a greedy algorithm, but as we will see in this survey, it is a difficult problem from the perspective of distributed local algorithms. Note that a maximal independent set is not necessarily a maximum independent set, i.e., an independent set that maximises $|I|$; finding a maximum independent set is a classical NP-hard optimisation problem.

4.3. Matchings. A set of edges $M \subseteq E$ is a matching if the edges in $M$ do not share a node, that is, if $\{t, u\} \in M$ and $\{t, v\} \in M$ then $u=v$. Again, a matching is maximal if it cannot be extended. A set of edges $M \subseteq E$ is a simple 2-matching if for each node $u$, the number of edges $e \in M$ with $u \in e$ is at most 2 .

Matchings have applications that are similar to those of independent sets: they identify a conflict-free set of activities. For example, if an edge $\{u, v\} \in E$ represents a data transmission between the devices $u$ and $v$, and each device can take part in at most one data transmission at a time, then a matching identifies a set of data transmissions that can be active simultaneously [63].

Many distributed systems have a natural bipartite structure: for example, there are clients that are connected to servers, or mobile users that communicate with base stations. In such systems, a matching $M$ can be interpreted as an assignment: which server serves which client. 
If $M$ is a matching in a bipartite graph, we say that an edge $\{u, v\} \in E \backslash M$ is unstable if $\{u, s\} \in M$ implies $p(u, s)>p(u, v)$ and $\{v, t\} \in M$ implies $p(v, t)>p(v, u)$. That is, if we interpret port numbers as a ranking of possible partners, both $u$ and $v$ would prefer each other to their current partners (if any). Matching $M$ is $\varepsilon$-stable [48] if the number of unstable edges is at most $\varepsilon|M|$, and the matching is stable [54,62] if there is no unstable edge. Stable matchings are attractive if servers and clients are selfish agents: if a matching is stable, then there is no client-server pair that would prefer to unilaterally change the matching in their own benefit.

We can also study relaxations of matchings. Let $\mathcal{G}$ be a bipartite graph with the parts $V=V_{1} \cup V_{2}$. Assume that $M \subseteq E$ is a subset of edges, and let $\operatorname{deg}_{M}(v)=|\{e \in M: v \in e\}|$ be the number of edges in $M$ that are incident to $v \in V$. We say that $M$ is a semi-matching [66] if $\operatorname{deg}_{M}(u)=1$ for all $u \in V_{1}$. Intuitively, a semi-matching assigns each client $u \in V_{1}$ to exactly one server $v \in V_{2}$, but a single server may receive multiple requests. Now if a server $v \in V_{2}$ processes the requests sequentially, the first request is handled in 1 time unit, the second request is handled in 2 time units, etc. Let $c(M, v)=1+2+\cdots+\operatorname{deg}_{M}(v)$ be the total processing delay of the tasks that are handled by the server $v \in V_{2}$, and let $c(M)=\sum_{v} c(M, v)$ be the total processing delay of all tasks. An aptimal semi-matching $M$ minimises the cost function $c(M)$; intuitively, and optimal semi-matching balances the load as equally as possible, in order to minimise the average waiting times of the clients.

4.4. Domination and covers. A set of nodes $D \subseteq V$ is a dominating set if every node in $V \backslash D$ has a neighbour in $D$. A dominating set $D$ is connected if the subgraph of $\mathcal{G}$ induced by $D$ is connected.

Applications of dominating sets can be found, for example, in wireless sensor networks [90]. In such networks, we can interpret $\mathcal{G}$ as a redundancy graph: an edge $\{u, v\} \in E$ indicates that the sensor nodes $u$ and $v$ are so close to each other that they are pairwise redundant - whenever device $u$ is active, device $v$ can be asleep and vice versa [22]. Hence if $D$ is a dominating set, then all other devices $V \backslash D$ can be asleep and conserve energy.

There are many problems that are related to dominating sets. We will here focus on three examples: edge dominating sets, edge covers, and vertex covers.

A set of edges $D \subseteq E$ is an edge dominating set $[24,52,151]$ if for each edge $\{u, v\} \in E \backslash D$ there is an edge $e \in D$ incident to $u$ or $v$ or both. Edge dominating sets are closely related to matchings: a maximal matching is an edge dominating set, and a minimum-size maximal matching is a minimum-size edge dominating set $[2,151]$. The connection between dominating sets and independent sets is weaker: a maximal independent set is a dominating set, but a minimum-size maximal independent set is not necessarily a minimum-size dominating set.

A set of edges $C \subseteq E$ is an edge cover if for each node $v \in V$ there is an edge $e \in C$ with $v \in e$. An edge cover exists if and only if there are no isolated nodes. A set of nodes $C \subseteq V$ is a vertex cover if $V \backslash C$ is an independent set. In other words, $C$ is a vertex cover if for each edge $\{u, v\} \in E$ either $u \in C$ or $v \in C$ or both. 


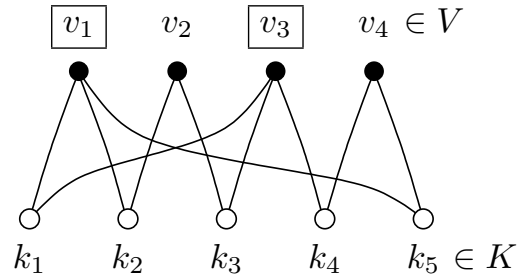

(a)

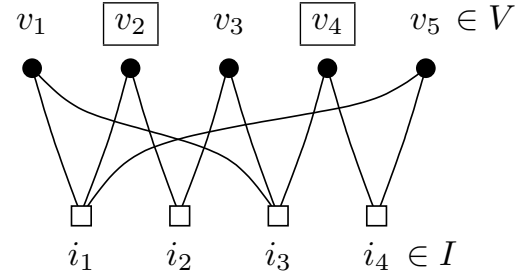

(b)

Figure 2. (a) A set cover instance with $\Delta_{V}=3$ and $\Delta_{K}=2$; a minimum-size solution is $X=\left\{v_{1}, v_{3}\right\}$. (b) A set packing instance with $\Delta_{V}=2$ and $\Delta_{I}=3$; a maximum-size solution is $X=\left\{v_{2}, v_{4}\right\}$.

4.5. Partitions. A domatic partition [25, 40] is a partition of $V$ into disjoint dominating sets. A domatic partition $V=D_{1} \cup D_{2} \cup \cdots \cup D_{k}$ can be interpreted as a schedule that controls a wireless sensor network [22]: first we activate all nodes in $D_{1}$ and put all other nodes asleep, then we activate all nodes in $D_{2}$ and put all other nodes asleep, etc.

A vertex $k$-colouring of $\mathcal{G}$ assigns a colour from the set $\{1,2, \ldots, k\}$ to each node of $\mathcal{G}$ such that adjacent nodes have different colours. An edge colouring is analogous: one assigns a colour to each edge such that adjacent edges have different colours. Put otherwise, a vertex colouring partitions $V$ into disjoint independent sets, and and edge colouring partitions $E$ into disjoint matchings; such partitions can be used to schedule the activities of nodes and edges in a conflict-free manner.

Naor and Stockmeyer [121] define the problem of weak colouring. A weak $k$-colouring of $\mathcal{G}$ assigns labels $\{1,2, \ldots, k\}$ to the nodes of $\mathcal{G}$ such that each non-isolated node has at least one neighbour with a different label. Any graph admits a weak 2-colouring. Note that weak colouring is related to domatic partitions: if there are no isolated nodes, then a weak 2-colouring is also a domatic partition of size 2 .

A cut is an arbitrary partition of $V$ into two sets, $V=X \cup Y$. The size of the cut is the number of edges $\{u, v\} \in E$ with $u \in X$ and $v \in Y$.

4.6. Covering problems. Let $\mathcal{G}=(V \cup K, E)$ be a bipartite graph. Each edge $\{v, k\} \in E$ joins an agent $v \in V$ and a customer $k \in K$; each node knows its role. The maximum degree of an agent $v \in V$ is $\Delta_{V}$, and the maximum degree of a customer $k \in K$ is $\Delta_{K}$. A subset $X \subseteq V$ is a set cover if each customer is covered by at least one agent in $X$, that is, for each customer $k \in K$ there is an adjacent agent $v \in X$ with $\{k, v\} \in E$. See Figure 2a.

The vertex cover problem is a special case of the set cover problem with $\Delta_{K}=2$ : each customer (edge) can be covered by 2 agents (nodes). The edge cover problem is a special case of the set cover problem with $\Delta_{V}=2$ : each agent (edge) covers 2 customers (nodes). The dominating set problem in a graph with maximum degree $\Delta$ is a special case of the set cover problem with $\Delta_{V}=\Delta_{K}=\Delta+1$. 
The problem of finding a minimum-size set cover can be written as an integer program

$$
\begin{aligned}
& \text { minimise } \sum_{v \in V} x_{v} \\
& \text { subject to } \sum_{v \in V} c_{k v} x_{v} \geq 1 \quad \forall k \in K \text {, } \\
& x_{v} \in\{0,1\} \quad \forall v \in V,
\end{aligned}
$$

where $c_{k v}=0$ if $\{k, v\} \notin E$ and $c_{k v}=1$ if $\{k, v\} \in E$. The LP relaxation of (1) is a $0 / 1$ covering $L P$

$$
\begin{aligned}
& \operatorname{minimise} \sum_{v \in V} x_{v} \\
& \text { subject to } \sum_{v \in V} c_{k v} x_{v} \geq 1 \quad \forall k \in K, \\
& x_{v} \geq 0 \quad \forall v \in V .
\end{aligned}
$$

In a general covering $L P$ we can have an arbitrary $c_{k v} \geq 0$ for each edge $\{k, v\} \in E$.

4.7. Packing problems. Let $\mathcal{G}=(V \cup I, E)$ be a bipartite graph. Each edge $\{v, i\} \in E$ joins an agent $v \in V$ and a constraint $i \in I$; each node knows its role. The maximum degree of an agent $v \in V$ is $\Delta_{V}$, and the maximum degree of a constraint $i \in I$ is $\Delta_{I}$. A subset $X \subseteq V$ is a set packing if each constraint is covered by at most one agent in $X$, that is, for each constraint $i \in I$ there is at most one adjacent agent $v \in X$ with $\{v, i\} \in E$. See Figure 2b.

The independent set problem is a special case of the set packing problem with $\Delta_{I}=2$. The maximum matching problem is a special case of the set packing problem with $\Delta_{V}=2$.

The problem of finding a maximum-size set packing can be written as an integer program

$$
\begin{aligned}
\operatorname{maximise} & \sum_{v \in V} x_{v} \\
\text { subject to } \sum_{v \in V} a_{i v} x_{v} \leq 1 & \forall i \in I, \\
x_{v} \in\{0,1\} & \forall v \in V,
\end{aligned}
$$

where $a_{i v}=0$ if $\{i, v\} \notin E$ and $a_{i v}=1$ if $\{i, v\} \in E$. The LP relaxation of (3) is a $0 / 1$ packing $L P$

$$
\begin{aligned}
& \text { maximise } \sum_{v \in V} x_{v} \\
& \text { subject to } \sum_{v \in V} a_{i v} x_{v} \leq 1 \quad \forall i \in I, \\
& x_{v} \geq 0 \quad \forall v \in V .
\end{aligned}
$$

In a general packing $L P$ we can have an arbitrary $a_{i v} \geq 0$ for each $\{i, v\} \in E$. A packing LP is a dual of a covering LP and vice versa. 
4.8. Mixed packing and covering. Finally, we can study linear programs with both packing constraints (constraints of the form $A \mathrm{x} \leq \mathbf{1}$ for a nonnegative matrix $A$ ) and covering constraints (constraints of the form $C \mathbf{x} \geq \mathbf{1}$ for a non-negative matrix $C$ ). In general, it may be that there is no feasible solution that satisfies both packing and covering constraints; however, we can formulate a linear program where the objective is to violate the covering constraints as little as possible (the case of violating the packing constraints as little as possible is analogous). We arrive at a max-min $L P$ where the objective is to maximise $\omega$ subject to $A \mathbf{x} \leq \mathbf{1}, C \mathbf{x} \geq \omega \mathbf{1}$, and $\mathbf{x} \geq \mathbf{0}$.

In a distributed setting, we have a bipartite graph $\mathcal{G}=(V \cup I \cup K, E)$. Each edge $e \in E$ is of the form $e=\{v, i\}$ or $e=\{v, k\}$ where $v \in V$ is an agent, $i \in I$ is a constraint, and $k \in K$ is a customer (or an objective); each node knows its role. The maximum degree of an agent $v \in V$ is $\Delta_{V}$, the maximum degree of a constraint $i \in I$ is $\Delta_{I}$, and the maximum degree of a customer $k \in K$ is $\Delta_{K}$. The objective is to

$$
\begin{array}{rlrl}
\text { maximise } & \omega & \\
\text { subject to } & \sum_{v \in V} a_{i v} x_{v} \leq 1 & \forall i \in I, \\
\sum_{v \in V} c_{k v} x_{v} \geq \omega & \forall k \in K, \\
x_{v} \geq 0 & \forall v \in V .
\end{array}
$$

Again, $a_{i v}=0$ if $\{i, v\} \notin E, a_{i v} \geq 0$ if $\{i, v\} \in E, c_{k v}=0$ if $\{k, v\} \notin E$, and $c_{k v} \geq 0$ if $\{k, v\} \in E$. In a $0 / 1 \max$-min $L P$ we have $a_{i v}, c_{k v} \in\{0,1\}$ for all $i \in I, k \in K$, and $v \in V$.

The applications of max-min LPs and analogous min-max LPs include tasks related to fair bandwidth allocation in communication networks and lifetime maximisation in wireless sensor networks [42].

\section{Auxiliary information and LOCAL VieWs}

In the model defined in Section 2, we have not assumed that the local algorithm has access to any information beyond the port numbering and the task-specific local input $i_{v}$. If we do not have any auxiliary information such as unique node identifiers in $i_{v}$, we call the network anonymous. In this section we will explore the fundamental limitations of anonymous networks, and possible extensions of the model.

5.1. Symmetry breaking. In an anonymous network, a port numbering does not provide enough information to break the symmetry $[5,80,150]$. To see this, consider a deterministic local algorithm and the network in Figure 1a on page 3 . The port-numbered graph is symmetric. It is easy to see that we cannot break the symmetry with the local algorithm if the local inputs are identical. Whatever message node $u$ sends to its port $x \in\{1,2\}$ on the first communication round, node $v$ sends the same message to its port $x$ if both run the same deterministic algorithm. Whatever message node $u$ receives from its port $x$ on the first communication round, node $v$ receives the same message from its port $x$. The local state of node $u$ after $r$ communication 

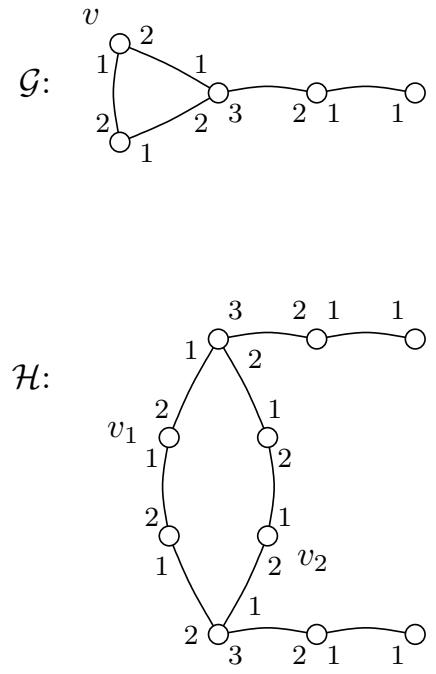

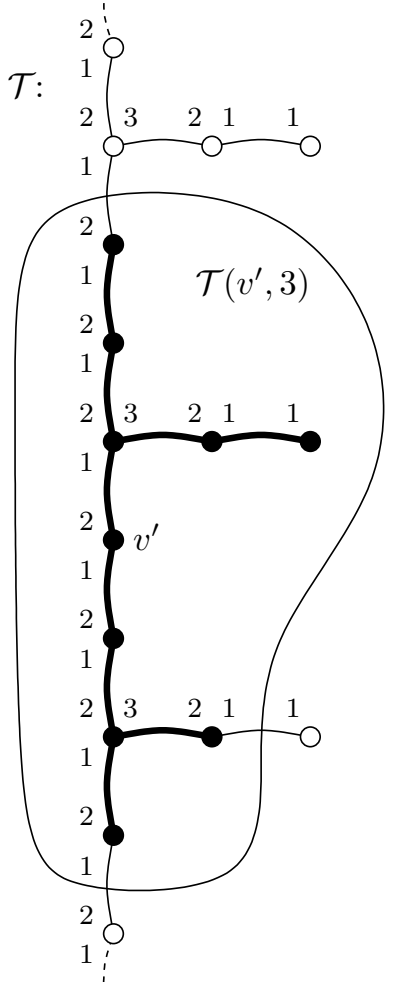

Figure 3. Covering graphs.

rounds is equal to the local state of node $v$ after $r$ communication rounds. Eventually, the local output $o_{u}$ is identical to the local output $o_{v}$.

More generally, we can choose the port numbers in an $n$-cycle so that for each node the port number 1 leads in a counterclockwise direction and the port number 2 leads in a clockwise direction. If the local inputs are identical, the local outputs are identical as well, regardless of the local horizon $r$. From the point of view of most combinatorial problems, this is discouraging: an empty set is the only matching or independent set that can be constructed by any local algorithm in this case; the set of all nodes is the only dominating set or vertex cover that can be constructed; and vertex colouring or edge colouring is not possible.

However, there are some positive examples of local algorithms that do not require any auxiliary information besides a port numbering. To better understand the possibilities and limitations of this model, we first introduce the concepts of covering graphs and unfoldings.

5.2. Covering graphs and unfoldings. We say that a port-numbered graph $\mathcal{H}=\left(V_{\mathcal{H}}, E_{\mathcal{H}}\right)$ is a covering graph of $\mathcal{G}=(V, E)$ if there is a surjective mapping $f: V_{\mathcal{H}} \rightarrow V$ with the following property: for each $v \in V_{\mathcal{H}}$ and for each integer $x$, the neighbour $x$ of $v$ in $\mathcal{H}$ is $u$ if and only if the neighbour $x$ of $f(v)$ in $\mathcal{G}$ is $f(u)$. The surjection $f$ is a covering map. See Figure 3 for an illustration: graph $\mathcal{H}$ is a covering graph of $\mathcal{G}$; we can choose a covering map $f$ with $f\left(v_{1}\right)=f\left(v_{2}\right)=v$. 
The unfolding or the universal covering graph [5] of a connected graph $\mathcal{G}$ is an acyclic, connected covering graph $\mathcal{T}$. The unfolding always exists, it is unique (up to isomorphism), and it is finite if and only if $\mathcal{G}$ is a tree. See Figure 3 for an illustration: the infinite tree $\mathcal{T}$ is the unfolding of $\mathcal{G}$; we can choose a covering map $f$ with $f\left(v^{\prime}\right)=v$. The tree $\mathcal{T}$ is also the unfolding of $\mathcal{H}$; we can choose, for example, a covering map $f$ with $f\left(v^{\prime}\right)=v_{1}$. This is no coincidence; because $\mathcal{H}$ and $\mathcal{G}$ have a common covering graph - in this case $\mathcal{H}$ - they also have the same unfolding.

Informally, we can construct the unfolding $\mathcal{T}$ of a graph $\mathcal{G}$ as follows. Choose an arbitrary node of $\mathcal{G}$ as a starting point. Traverse graph $\mathcal{G}$ in a breadth-first manner; if we revisit a node because of a cycle, treat it as a new node.

This simple intuitive explanation of the unfolding is sufficient for our purposes. See, e.g., Godsil and Royle [58, §6.8] for more information on covering graphs in a pure graph-theoretic setting; note that the term "lift" has also been used to refer to a covering graph $[4,73]$. For more information on universal covering graphs, see, e.g., Angluin [5]. An analogous concept in topology is a universal covering space, see, e.g., Hocking and Young [71, $\S 4.8]$ or Munkres [119, §80].

5.3. Local view. Let $v$ be a node in an anonymous, port-numbered network $\mathcal{G}$. Let $\mathcal{T}$ be the unfolding of $\mathcal{G}$, and let $v^{\prime}$ be a preimage of $v$ in the covering map $f$, as in the example of Figure 3.

The radius-r local view of node $v$ is the subgraph $\mathcal{T}\left(v^{\prime}, r\right)$ of $\mathcal{T}$ induced by $B_{\mathcal{T}}\left(v^{\prime}, r\right)$. Put otherwise, the radius- $r$ local view of $v$ is the radius- $r$ neighbourhood of its preimage $v^{\prime}$ in the unfolding. See Figure 3 for an illustration in the case $r=3$. The local view does not depend on the choice of $v^{\prime} \in f^{-1}(\{v\})$.

Now we are ready to characterise exactly what we can do in the port numbering model. We begin with the good news. In a deterministic local algorithm with local horizon $r$, each node $v$ can construct its radius- $r$ local view $[19,150]$. There is a simple local algorithm that gathers this information in $r$ communication rounds: Initially, each node knows its radius-0 local view. In communication round $i$, each node floods its radius- $(i-1)$ local view to each neighbour, and includes the outgoing port number in the message. After round $i$, each node pieces together the local views received from its neighbours; this results in the radius- $i$ local view. We can also gather the local input for each node in the local view. Hence, in a local algorithm each node $v$ can choose its output $o_{v}$ based on all information that is available in its radius- $r$ local view. If the local views of nodes $u$ and $v$ differ, then the local outputs of nodes $u$ and $v$ can differ as well.

The bad news is that choosing the local output based on the local view is, in a sense, the only thing that one can do in a local algorithm $[5,19,150]$. This is easily understood if we consider the covering map $f$ from the unfolding $\mathcal{T}$ to the communication graph $\mathcal{G}$, and apply the same local algorithm $\mathcal{A}$ in both $\mathcal{T}$ and $\mathcal{G}$. Initially, for each node $v^{\prime}$ in $\mathcal{T}$, the local state of $v^{\prime}$ in $\mathcal{T}$ and $v=f\left(v^{\prime}\right)$ in $\mathcal{G}$ is the same. Furthermore, on each communication round, $v^{\prime}$ and $v$ perform the same local computation, send the same messages, and receive the same messages - here we use the fact that $f$ is a local isomorphism 


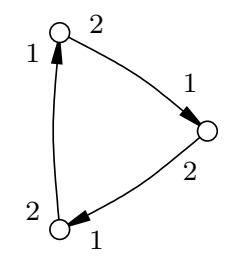

Figure 4. A communication graph with a port numbering and an orientation; cf. Figure 1.

that preserves the port numbering. Hence, after $r$ communication rounds, both $v^{\prime}$ and $v$ must produce the same output. Therefore the output of $v$ in a local algorithm with local horizon $r$ only depends on its local view $B_{\mathcal{T}}\left(v^{\prime}, r\right)$.

Among others, this shows that a local algorithm cannot distinguish between $\mathcal{G}$ and $\mathcal{H}$ in Figure 3 because they have the same unfolding $\mathcal{T}$. Node $v$ in $\mathcal{G}$, nodes $v_{1}$ and $v_{2}$ in $\mathcal{H}$, and node $v^{\prime}$ in $\mathcal{T}$ all produce the same output. We can see that a local algorithm in an anonymous network cannot even detect if there are triangles (3-cycles) in the network.

5.4. Graphs with orientation. So far we have assumed that we have a port numbering in the communication graph $\mathcal{G}$. We proceed to study a slightly stronger assumption [115]: in addition to the port numbering, we are given an orientation of graph $\mathcal{G}$. That is, for each edge $\{u, v\} \in E$, we have chosen exactly one direction, either $(u, v)$ or $(v, u)$. See Figure 4 for an illustration. In a port numbering, each node chooses an ordering on incident edges, while in an orientation, each edge chooses an ordering on incident nodes.

We use the standard terminology for directed graphs: If the edge $\{u, v\}$ has the orientation $(u, v)$, then $u$ is a predecessor of $v$ and $v$ is a successor of $u$. The in-degree of a node is the number of predecessors, i.e., the number of edges entering the node. Similarly, the out-degree of a node is the number of successors, i.e., the number of edges leaving the node.

At first sight, having an arbitrary orientation in addition to an arbitrary port numbering does not seem to help much. In an $n$-cycle, we can have all edges directed consistently in a clockwise direction, as shown in Figure 4. Hence we obtain the same negative results as in the case of an $n$-cycle with only a port numbering. More generally, we can construct a $d$-regular graph for any even constant $d$ such that the local view of each node is identical [121], in spite of a port numbering and an orientation; see Figure 5 for an example. Furthermore, as shown in Figure 6, having an orientation does not help one to tell a graph from its cover.

Surprisingly, it turns out that in graphs where every node has an odd degree, an orientation together with a port numbering is enough to break the symmetry in the following sense: the output of a non-isolated node $v$ is different from the output of at least one neighbour $u$ of $v$.

Example 2. Consider a 3-regular graph with a port numbering and an orientation. Let $v$ be an arbitrary node; we show that the local view of $v$ is different from the local view of at least one neighbour of $v$. Figure 7 illustrates the three possible cases. In Figure 7a, node $v$ and its neighbour $u$ 


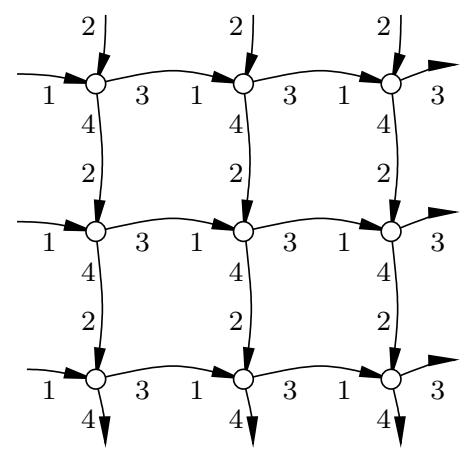

FiguRE 5. A 4-regular graph with a port numbering and an orientation.

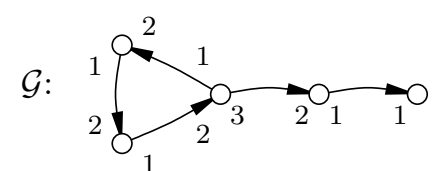

$\mathcal{H}:$

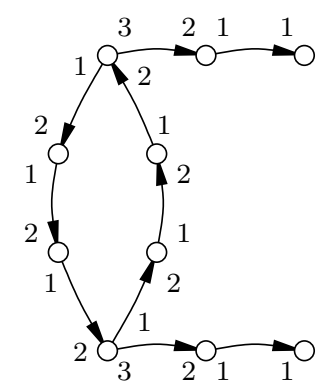

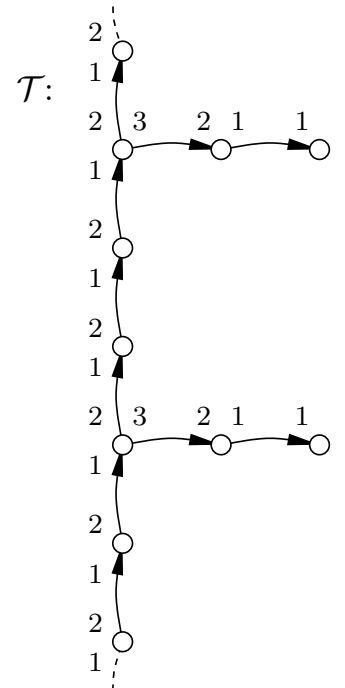

FiguRE 6. Covering graphs with a port numbering and an orientation; cf. Figure 3.

have different out-degrees; hence the local view of $v$ differs from the local view of $u$. Otherwise the out-degree of $v$ and each neighbour of $v$ is the same. The common out-degree of $v$ and its neighbours is either 1 or 2. Figure $7 \mathrm{~b}$ illustrates the case where the common out-degree is 2 . In this case the local view of $s$ is necessarily different from the local view of $t$ : both have exactly one predecessor, and the port numbers assigned to these unique incoming edges are different because $p(v, s) \neq p(v, t)$. Therefore the local view of $v$ is different from the local view of $s$ or $t$ (or both). Figure 7c illustrates the case where the common out-degree is 1 ; this is analogous to the case of the out-degree 2.

This argument can be generalised to any graph, as long as the degree of each node is odd. We present the details in Section 7.3 when we review a local algorithm for weak colouring $[115,121]$.

5.5. Graphs with unique identifiers. We can make an even stronger assumption: each node $v$ has a globally unique identifier as part of its 


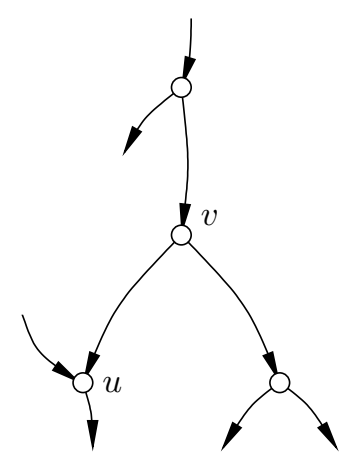

(a)

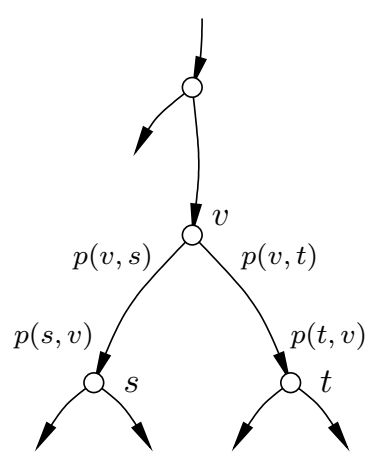

(b)

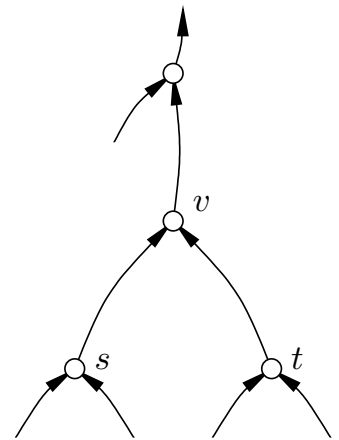

(c)

FiguRE 7. A 3-regular graph with a port numbering and an orientation. (a) Different out-degrees. (b) Out-degree is 2. (c) Out-degree is 1 .

local input $i_{v}$. Usually the identifiers are assumed to be a permutation of $\{1,2, \ldots,|V|\}$ or a subset of $\{1,2, \ldots$, poly $(|V|)\}$. Naturally we require that the local algorithm solves the problem for any choice of unique identifiers.

Globally unique identifiers are a standard assumption in the field of local algorithms. This is a strictly stronger assumption than having only port numbers and an orientation available; all negative results for the case of globally unique identifiers imply negative results in anonymous networks and anonymous oriented networks.

Unfortunately, the assumption on globally unique identifiers means that the problem is not of distributed constant size: the number of bits required to encode the identifiers increases as the size of the network increases. In practice, for many algorithms that are designed under the assumption of globally unique identifiers, it is sufficient to have locally unique identifiers. That is, we assume that a local algorithm with local horizon $r$ has access to identifiers that are unique within every radius- $r$ neighbourhood in the communication graph $\mathcal{G}$. In a bounded-degree graph, it is then possible to choose identifiers that are locally unique but have constant size.

With globally or locally unique identifiers, a node $v$ in graph $\mathcal{G}$ can tell for each pair of nodes $s, t$ in its radius- $r$ local view whether $f(s)=f(t)$, that is, whether they represent the same node in the original graph $\mathcal{G}$. This implies that each node $v$ can reconstruct the subgraph $\mathcal{G}[v, r]$ of $\mathcal{G}$ - see Section 2.2 for the definition and Figure 8 for an illustration. Hence, when we study local algorithms in networks with unique identifiers, it is sufficient to present a function that maps the subgraph $\mathcal{G}[v, r]$ to the local output $o_{v}[121]$.

We note that the difference between $\mathcal{G}(v, r)$ and $\mathcal{G}[v, r]$ is usually immaterial. After all, $\mathcal{G}[v, r+1]$ contains $\mathcal{G}(v, r)$ as a subgraph, and $\mathcal{G}(v, r)$ contains $\mathcal{G}[v, r]$ as a subgraph. We are typically not interested in additive constants in the local horizon $r$. Hence we can use either $\mathcal{G}(v, r)$ or $\mathcal{G}[v, r]$ to derive both positive and negative results, whichever is more convenient. If the local output cannot be determined based on $\mathcal{G}(v, r)$ for any constant $r$, then there is no local algorithm for the task; if it can be determined based on $\mathcal{G}(v, r)$ for some constant $r$, then there is a local algorithm. We can even go as far 


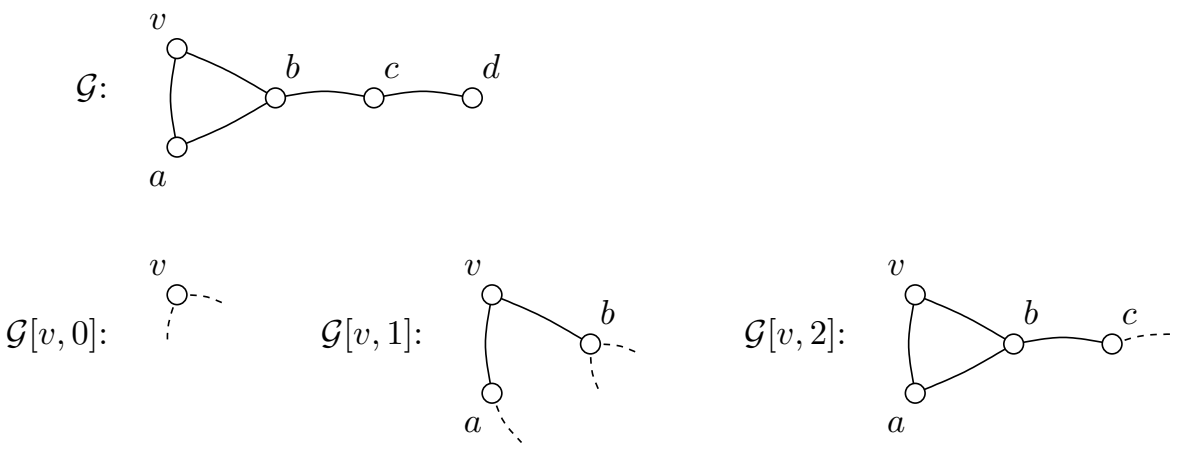

Figure 8. The local view of node $v$ in a graph $\mathcal{G}$ with unique node identifiers.

as to use this as the definition of a local algorithm, if we study networks with unique identifiers.

\section{Negative Results}

In this section we review negative results for local algorithms. Non-trivial results are summarised in Tables 1 and 2 .

6.1. Preliminary observations. There are two simple arguments that can be used to show that a problem cannot have a local algorithm: inherently non-local problems and the impossibility of symmetry-breaking.

A problem is inherently non-local if the output at a node $u$ may depend on the input at a node $v$ with $d_{\mathcal{G}}(u, v)=\Omega(|V|)$. By definition, a local algorithm cannot solve a problem that is inherently non-local. Constructing a spanning tree is a classical example of a simple problem that is inherently non-local; see Figure 9 for an illustration. Finding a stable matching is another example of a non-local problem [48].

The problem of finding a maximal matching is, in a sense, much more local. For example, if we already have a solution $M$ for a graph $\mathcal{G}$, a local change in $\mathcal{G}$ requires only local changes in the solution $M$. However, as we discussed in Section 5.1, it is not possible to break the symmetry with a local algorithm in an $n$-cycle if the nodes are anonymous; a port numbering and an orientation of $\mathcal{G}$ do not help. Therefore it is not possible to find a maximal matching in an anonymous $n$-cycle, oriented or not. The same negative results apply to the problems of finding a maximal independent set, a vertex colouring, an edge colouring, a weak colouring, an $O(1)$-approximation of a maximum matching, an $O(1)$-approximation of a maximum independent set, a $(3-\varepsilon)$-approximation of a minimum dominating set, and a $(2-\varepsilon)$-approximation of a minimum vertex cover in anonymous $n$-cycles. As a straightforward generalisation, there is no local $o(\Delta)$-approximation algorithm for the minimum dominating set problem in anonymous bounded-degree graphs.

In what follows, we focus on negative results that hold even if globally unique identifiers are available. 
TABle 1. Problems that cannot be solved with a deterministic local algorithm, even if there are unique node identifiers. The problems are defined in Section 4.

\begin{tabular}{lll}
\hline Problem & Graph family & References \\
\hline Maximal independent set & cycle & {$[109]$} \\
Maximal matching & cycle & {$[109]$ cor. } \\
Vertex 3-colouring & cycle & {$[109]$} \\
Vertex $\Delta$-colouring & $(\Delta+1)$-coloured tree & {$[91]$} \\
Edge colouring & cycle & {$[109]$ cor. } \\
Weak colouring & $2 k$-regular & {$[121]$} \\
\hline
\end{tabular}

cor. $=$ corollary, see text

TABLE 2. Approximation factors that cannot be achieved with a deterministic local algorithm, even if there are unique node identifiers.

\begin{tabular}{llll}
\hline Problem & $\begin{array}{l}\text { Approx. } \\
\text { factor }\end{array}$ & Graph family & References \\
\hline Independent set & $O(1)$ & cycle & {$[30,103,106]$} \\
Matching & $O(1)$ & general & {$[91,98,117]$} \\
& $O(1)$ & cycle & {$[30]$} \\
Edge cover & $2-\varepsilon$ & cycle & {$[30,103,106]$ cor. } \\
Vertex cover & $O(1)$ & general & {$[91,95,100,117]$} \\
& $2-\varepsilon$ & cycle & {$[30,103,106]$ cor. } \\
Dominating set & $O(1)$ & general & {$[91,95,100,117]$} \\
& $O(1)$ & unit-disk & {$[103,106]$} \\
& $2 k+1-\varepsilon$ & $2 k$-regular & {$[30]$ cor. } \\
& $k+1-\varepsilon$ & $(2 k+1)$-regular, 2 -C & {$[8]$} \\
& $5-\varepsilon$ & 4 -regular, planar & {$[30]$} \\
Domatic partition & $3-\varepsilon$ & cycle & {$[30,103,106]$} \\
Edge domin. set & $3-\varepsilon$ & cycle & {$[30,103,106]$ cor. } \\
Maximum cut & $O(1)$ & cycle & {$[30,103,106]$ cor. } \\
\hline Set cover & $k-\varepsilon$ & $k$-regular & {$[30,103,106]$ cor. } \\
\hline 0/1 packing LP & $O(1)$ & general & {$[9,30,103,106]$} \\
0/1 covering LP & $O(1)$ & general & {$[91,98,117]$} \\
0/1 max-min LP & $\alpha$ & bounded-degree & {$[91,95,100,117]$} \\
\hline$\varepsilon>0, \alpha=\Delta_{I}\left(1-1 / \Delta_{K}\right)$ & & {$[42,44,46]$} \\
\hline 2-C $=$ bicoloured graphs, i.e., a 2 2-colouring is given & \\
cor. $=$ corollary, see text & & \\
& & &
\end{tabular}


(a)

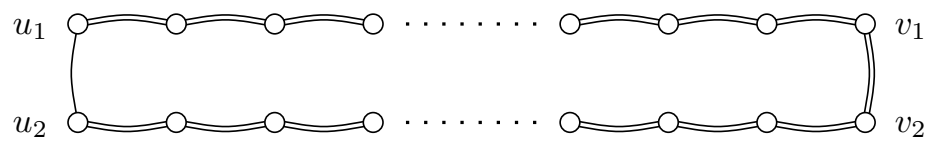

(b)

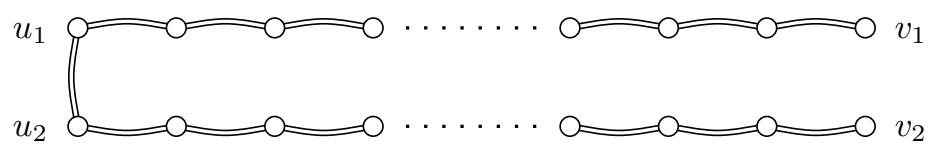

(c)

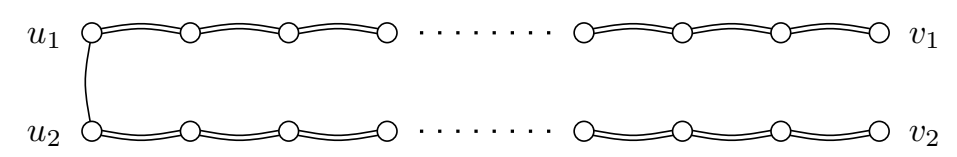

FiguRE 9 . (a) An $n$-cycle $\mathcal{G}$; the double lines show a spanning tree. (b) A local change in graph $\mathcal{G}$ near nodes $v_{1}$ and $v_{2}$ requires a non-local change in the spanning tree near nodes $u_{1}$ and $u_{2}$. (c) A local algorithm cannot even verify whether a given set of edges is a spanning tree or not [84]. In every local neighbourhood, this non-tree looks similar to a spanning tree in part (a) or (b).

6.2. Comparable identifiers. Let us first focus on order-invariant algorithms: we assume that unique node identifiers are available, but the algorithm is only allowed to compare the identifiers and not access their numerical value.

It turns out that being able to compare identifiers does not help much in symmetry breaking. For example, in an $n$-cycle, we can assign the node identifiers $1,2, \ldots, n$ in an increasing order. If we pick any two nodes $u, v \in U=\{r+1, r+2, \ldots, n-r\}$, then the radius- $r$ neighbourhood of $u$ looks identical to the radius- $r$ neighbourhood of $v$, assuming that a node can only exploit the ordering of the identifiers. Therefore every node in $U$ must make the same decision; see, e.g., Kuhn [91, §2.7.2] and Floréen et al. [47]. We immediately obtain the same negative results that we had for anonymous networks in an $n$-cycle: for example, there is no local algorithm for finding a maximal matching, a maximal independent set, a vertex colouring, an edge colouring, or a weak colouring.

6.3. Numerical identifiers. A natural approach would be to exploit the numerical values of the identifiers; after all, this is exactly what the classical (distributed but not constant-time) algorithm for vertex colouring by Cole and Vishkin [26] does.

Unfortunately, a general result by Naor and Stockmeyer [121] shows that local algorithms for so-called locally checkable labellings - these include vertex colourings and maximal independent sets in bounded-degree graphs do not benefit from the numerical values of the identifiers: if there is a local algorithm that uses the numerical values, there is an order-invariant local algorithm as well.

More specifically, Linial [109] shows that a synchronous distributed algorithm for vertex 3 -colouring in an $n$-cycle with unique identifiers requires 
$\Omega\left(\log ^{*} n\right)$ communication rounds. Here $\log ^{*} n$ denotes the iterated (base-2) logarithm of $n$, that is, the smallest integer $k \geq 0$ such that $k$ iterated applications of the function $x \mapsto \log _{2} x$ to the initial value $n$ results in a value at most 1 .

Linial's result holds even under the assumption that there is a consistent clockwise orientation in the $n$-cycle. As a direct implication, an algorithm for finding an edge 3-colouring, a maximal independent set, or a maximal matching in an $n$-cycle requires $\Omega\left(\log ^{*} n\right)$ communication rounds as well. The barrier of $\Omega\left(\log ^{*} n\right)$ is hard to break even if we are allowed to provide arbitrary instance-specific advice to some nodes in the network [50].

6.4. Approximations for combinatorial problems. So far we have seen that there are no local algorithms for problems such as vertex colouring, edge colouring, maximal independent set, or maximal matching. However, it is possible to find a feasible independent set or a matching with a local algorithm (the empty set), and similarly there is a trivial local algorithm for finding a vertex cover or a dominating set (the set of all nodes). This raises the question of whether there is a local approximation algorithm for any of these problems, with a nontrivial approximation guarantee.

Unfortunately, this does not seem to be the case. Czygrinow et al. [30] and Lenzen and Wattenhofer [106], [103, §11] show that it is not possible to find a constant-factor approximation of a maximum independent set or a maximum matching in an $n$-cycle with a deterministic local algorithm. Czygrinow et al.'s elegant proof uses Ramsey's theorem [61, 129]; Lenzen and Wattenhofer build on Linial's [109] work.

These results imply that there is no local constant-factor approximation algorithm for the maximum cut problem in an $n$-cycle. If we can find a cut $\{X, Y\}$ of size $k$ in an $n$-cycle, then it is possible to find a matching with at least $k / 3$ edges as well. The edges that cross the cut form a bipartite graph $\mathcal{H}$, the cut $\{X, Y\}$ is a 2-colouring of the bipartite graph $\mathcal{H}$, and the algorithm that we will describe in Section 7.1 finds a maximal matching in the 2-coloured graph $\mathcal{H}$.

As another corollary, there is no local $(2-\varepsilon)$-approximation algorithm for the edge cover problem in a cycle. To see this, consider a $2 n$-cycle $\mathcal{G}=(V, E)$. A minimum edge cover has $n$ edges, and a maximum matching has $n$ edges as well. Hence a $(2-\varepsilon)$-approximate edge cover $C \subseteq E$ has at most $(2-\varepsilon) n$ edges, and its complement $M=E \backslash C$ has at least $\varepsilon n$ edges. Furthermore, each $v \in V$ is covered by at least one edge in $C$; therefore each $v \in V$ is covered by at most one edge in $M$. Hence $M$ is a matching, and within factor $1 / \varepsilon$ of the optimum.

An analogous argument shows that there is no local $(2-\varepsilon)$-approximation algorithm for the vertex cover problem in an $n$-cycle. Furthermore, there is no local $(3-\varepsilon)$-approximation algorithm for the dominating set problem. Note that the complement $X=V \backslash D$ of a dominating set $D$ in an $n$-cycle can be turned into an independent set [30]: a node $v \in X$ is adjacent to at most one other node $u \in X \backslash\{v\}$. Exchanging the roles of edges and nodes, the same argument shows that there is no local $(3-\varepsilon)$-approximation algorithm for the edge dominating set problem in a cycle. Moreover, we cannot find more than one disjoint dominating set in a cycle; because a 
a)

b)

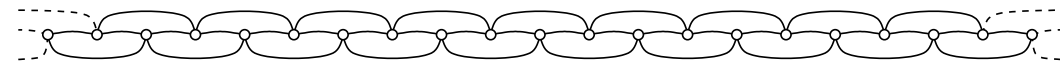

c)

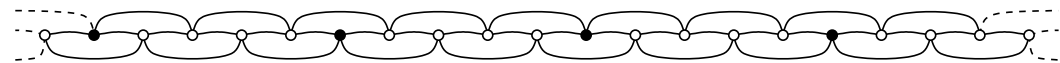

d)

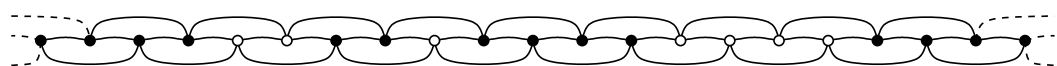

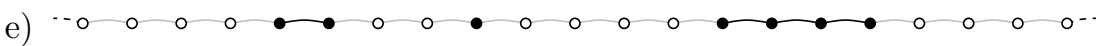

f) $\mathrm{Cr}^{\mathrm{O}}$

FiguRE 10 . There is no local $(2 k+1-\varepsilon)$-approximation algorithm for the dominating set problem in $2 k$-regular graphs (the case $k=2$ ).

$3 n$-cycle has 3 disjoint dominating sets, this shows that no local algorithm can find a $(3-\varepsilon)$-approximation of a maximum domatic partition.

More generally, Czygrinow et al. [30] and Lenzen and Wattenhofer [106] show that for any constant $\varepsilon>0$, a local algorithm cannot produce a factor $2 k+1-\varepsilon$ approximation of a minimum dominating set in $2 k$-regular graphs. The basic argument is as follows. Figure 10a shows a $(2 k+1) n$-cycle $\mathcal{G}=(V, E)$. Using a local algorithm, we can construct a $2 k$-regular graph $\mathcal{H}=\left(V, E^{\prime}\right)$, as illustrated in Figure 10b. A minimum dominating set of $\mathcal{H}$ has $n$ nodes (Figure 10c); therefore a hypothetical $(2 k+1-\varepsilon)$-approximation algorithm has to return a dominating set $D$ with at most $(2 k+1-\varepsilon) n$ nodes (Figure 10d). Therefore its complement $X=V \backslash D$ has at least $\varepsilon n$ nodes. Furthermore, because $D$ is a dominating set, there is no path with more than $2 k$ nodes in the subgraph of $\mathcal{G}$ induced by $X$ (Figure 10e). Hence we can construct an independent set $I$ with at least $\varepsilon n /(2 k)$ nodes (Figure 10f), which is a contradiction with the local inapproximability of the independent set problem in cycles.

Czygrinow et al. [30] consider the case $k=2$ to show that a local algorithm cannot find a factor $5-\varepsilon$ approximation of a minimum dominating set in planar graphs. Lenzen and Wattenhofer [106] consider a general $k$ to show that a local algorithm cannot find a constant-factor approximation of a minimum dominating set in unit-disk graphs (see Section 9.1 for the definition).

In the above proof, we have focused on regular graphs with an even degree. As we saw in Section 5.4, some amount of symmetry-breaking is possible in graphs where each node has an odd degree. Nevertheless, we can derive a slightly weaker result for regular graphs with an odd degree: a local algorithm cannot produce a factor $k+1-\varepsilon$ approximation of a minimum dominating set in $(2 k+1)$-regular graphs [8]. To see this, consider a $(k+1) n$-cycle $\mathcal{G}=(V, E)$; see Figure 11a. We can use a local algorithm to construct a $(2 k+1)$-regular graph $\mathcal{H}$ as illustrated in Figure 11b; each original node $v \in V$ simulates a pair of nodes, $v^{\prime}$ and $v^{\prime \prime}$, in $\mathcal{H}$. A minimum dominating set of $\mathcal{H}$ has $n$ nodes (Figure 11c). A hypothetical $(k+1-\varepsilon)$ approximation algorithm has to return a dominating set $D$ with at most 
a)

b)

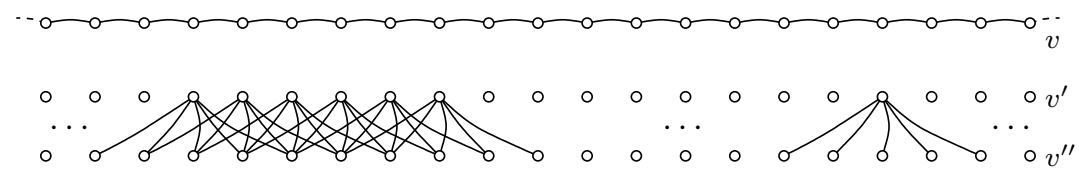

c)

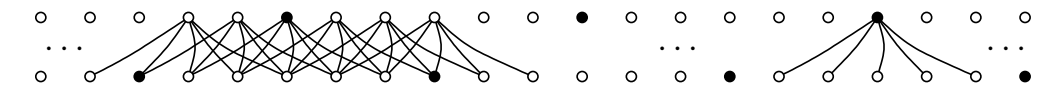

d)

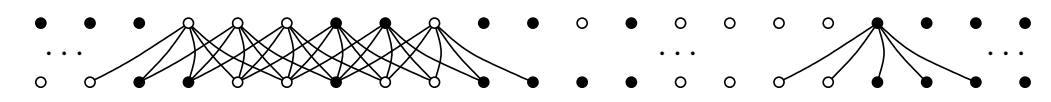

e) $\begin{array}{llllllllllllll}\cdots & 0 & 0 & 0 & \cdots & 0 & 0 & 0 & 0 & 0 & 0 & 0 & 0 & 0\end{array}$

f)

FIgURE 11. There is no local $(k+1-\varepsilon)$-approximation algorithm for the dominating set problem in $(2 k+1)$-regular graphs (the case $k=2$ ).

$(k+1-\varepsilon) n$ nodes (Figure 11d). Let $X \subseteq V$ consist of the nodes $v \in V$ such that both $v^{\prime} \notin D$ and $v^{\prime \prime} \notin D$ (Figure 11e). We know that the size of $X$ is at least $\varepsilon n$; furthermore, $X$ does not induce paths with more than $2 k$ nodes in $\mathcal{G}$. Hence we can construct an independent set $I$ with at least $\varepsilon n /(2 k)$ nodes (Figure 11f), a contradiction.

This negative result holds even in bipartite graphs where a 2-colouring is given as part of the local input. Incidentally, the construction in Figure 11 is the so-called bicoloured double cover of the construction in Figure 10; we will revisit bicoloured double covers in more detail when we present positive results in Section 7.1.

6.5. Approximations for LPs. The negative results in the previous section build on the impossibility of symmetry breaking in combinatorial problems. However, there are also negative results for linear programs.

Bartal et al. [18] observe that a $(1+\varepsilon)$-approximation algorithm for packing and covering LPs requires $\Omega(1 / \varepsilon)$ communication rounds.

Kuhn, Moscibroda, and Wattenhofer [91, 92, 95, 98, 100, 117] show that it is not possible to find a constant-factor approximation of a minimum vertex cover, minimum dominating set, or maximum matching in general graphs with a local algorithm, if there is no degree bound. The results extend to the LP relaxations of these problems as well, and hence to 0/1 packing LPs and $0 / 1$ covering LPs.

Floréen et al. [42, 44, 46] present a tight lower bound for the local approximability of max-min LPs. In bounded-degree graphs, no local algorithm can achieve the approximation factor $\Delta_{I}\left(1-1 / \Delta_{K}\right)$. 
TABLE 3. Deterministic local algorithms. The problems are defined in Section 4.

\begin{tabular}{llll}
\hline Problem & Graph family & Model & References \\
\hline Maximal matching & bicoloured, B-D & $\mathrm{P}$ & {$[64]$} \\
$\varepsilon$-stable matching & bicoloured, B-D & $\mathrm{P}$ & {$[48]$} \\
Vertex $(\Delta+1)$-colouring & $k$-coloured, B-D & $\mathrm{P}$ & {$[16,26,59,93]$} \\
Weak colouring & odd degree, B-D & $\mathrm{P}+\mathrm{O}$ & {$[115,121]$} \\
\hline
\end{tabular}

$\mathrm{B}-\mathrm{D}=$ bounded-degree graph

$\mathrm{P}=$ algorithm uses only a port numbering

$\mathrm{P}+\mathrm{O}=$ algorithm uses only a port numbering and an orientation

\section{Positive Results}

In spite of all negative results that we saw in Section 6, a few local algorithms are known. In this section, we review known deterministic local algorithms; prominent positive results are also summarised in Tables 3 and 4 .

We begin with two different techniques, both of which yield a local approximation algorithm for the vertex cover problem: Section 7.1 presents a technique based on bicoloured covering graphs; Section 7.2 presents a linear programming approach.

7.1. Bicoloured matchings and vertex covers. In a centralised setting, there is a simple 2-approximation algorithm for the vertex cover problem: find any maximal matching and take the endpoints. ${ }^{1}$ We cannot find a maximal matching with a local algorithm in general graphs; nevertheless, we can apply the same basic idea indirectly to design a local approximation algorithm for the vertex cover problem.

We say that the communication graph $\mathcal{G}$ is bicoloured if a vertex 2-colouring of $\mathcal{G}$ is given as part of the local input - each node knows whether it is black or white. Clearly graph $\mathcal{G}$ has to be bipartite; otherwise there is no 2-colouring.

Hańćkowiak et al. [64] present a simple local algorithm for the problem of finding a maximal matching in a bicoloured bounded-degree graph. A port numbering is enough; unique node identifiers are not needed. The algorithm performs the following two steps repeatedly:

(1) Each unmatched black node sends a proposal to one of its white neighbours. The neighbours are chosen in the order of port numbers.

(2) Each white node accepts the first proposal that it receives. If several proposals are received in the same round, ties are broken with port numbers.

After $2 \Delta$ steps, this results in a maximal matching $M$. To see that $M$ is maximal, consider an edge $e=\{u, v\} \in E \backslash M$ such that $u$ is a black node and $v$ is a white node. One of the following holds: (i) $u$ never sent a proposal to $v$, or (ii) $v$ rejected the proposal from $u$. In case (i), node $u$ is matched, and in case (ii), node $v$ is matched. Hence $M \cup\{e\}$ is not a matching.

\footnotetext{
${ }^{1}$ Papadimitriou and Steiglitz [123] attribute this algorithm to Fanica Gavril and Mihalis Yannakakis.
} 
TABLE 4. Deterministic local approximation algorithms. The algorithms without references are trivial; see text.

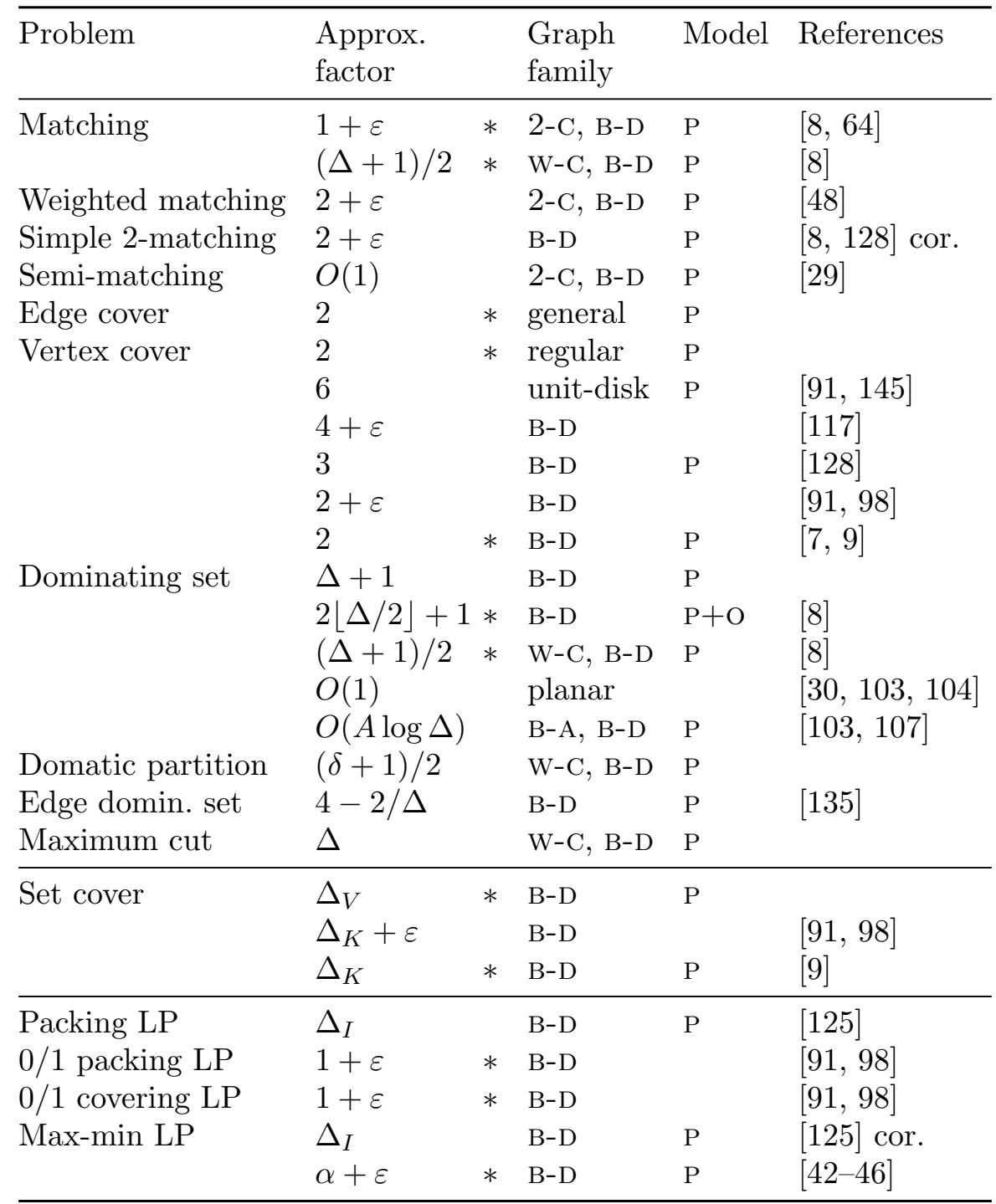

$\varepsilon>0, \quad \alpha=\Delta_{I}\left(1-1 / \Delta_{K}\right), \quad \delta=$ minimum degree of $\mathcal{G}$

$*=$ tight approximation ratio (matching negative result)

B-D $=$ graphs with node degrees at most $\Delta$

B-A $=$ graphs with arboricity $[34, \S 2.4]$ at most $A$

$2-\mathrm{C}=$ bicoloured graphs, i.e., a 2 -colouring is given

$\mathrm{W}-\mathrm{C}=\mathrm{a}$ weak 2 -colouring is given or can be found locally; includes graphs where every node has an odd degree [115, 121]

$\mathrm{P}=$ algorithm uses only a port numbering

$\mathrm{P}+\mathrm{O}=$ algorithm uses only a port numbering and an orientation

cor. = corollary, see text 
$\mathcal{G}: \overbrace{2}^{1} \int_{2}^{2} \overbrace{1}^{2} \overbrace{1}^{\circ}$

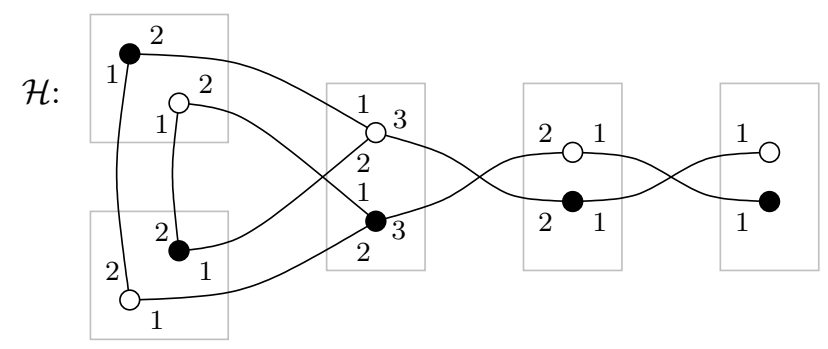

Figure 12. The bicoloured graph $\mathcal{H}$ is the bipartite double cover of graph $\mathcal{G}$. Note that the graphs are isomorphic to those in Figure 3.

Now we know how to find a maximal matching in a bicoloured graph. It turns out that with the help of this simple algorithm, it is possible to find a 3 -approximation of a minimum vertex cover in an arbitrary bounded-degree graph [128]. The key observation is the following: for any graph $\mathcal{G}$, we can construct the bicoloured graph $\mathcal{H}$ that is the bipartite double cover $[5,21,75]$ of $\mathcal{G}$; see Figure 12 for an illustration.

The bipartite double cover of $\mathcal{G}$, also known as the Kronecker double cover, is the Kronecker product [143] of the graphs $\mathcal{G}$ and $K_{2}$. In essence, for each original node $v$ in graph $\mathcal{G}$, we create two copies: a black copy and a white copy. If $u$ and $v$ are adjacent in the original graph $\mathcal{G}$, then the black copy of $u$ is adjacent to the white copy of $v$ in graph $\mathcal{H}$ and vice versa. Port numbers can be inherited from $\mathcal{G}$. It follows that $\mathcal{H}$ is a covering graph of $\mathcal{G}$ (see Section 5.2). Furthermore, it is a double cover: the covering map $f$ maps exactly 2 nodes of $\mathcal{H}$ onto each node of $\mathcal{G}$.

Now let $\mathcal{A}$ be the local algorithm for maximal matchings in bicoloured graphs. A local algorithm that runs in a general graph $\mathcal{G}$ can simulate the behaviour of $\mathcal{A}$ in the bicoloured double cover $\mathcal{H}$; a node $v$ in $\mathcal{G}$ is responsible for simulating the behaviour of both its black copy and its white copy. Once the simulation completes, each node can inspect the output produced by its two copies.

Hence we can find a maximal matching $M$ in the bicoloured double cover $\mathcal{H}$ and map it back to the original graph $\mathcal{G}$. This gives us a subset of edges $X \subseteq E$ in $\mathcal{G}=(V, E)$ with the following properties: (i) for each node $v \in V$, there are at most 2 edges incident to $v$ in $X$; and (ii) for each edge $\{u, v\} \in E$, at least one of $u, v$ is incident to an edge $x \in X$. Put otherwise, $X$ is a simple 2-matching and its endpoints are a vertex cover $C \subseteq V$.

A minimum-size vertex cover $C^{*}$ must cover all edges, including the edges in the simple 2-matching $X$. A node $v \in C^{*}$ can cover at most 2 edges in $X$, and an edge in $X$ is covered by at most 2 nodes in $C$. Hence $|C| \leq 2|X| \leq 4\left|C^{*}\right|$. We have a simple algorithm that finds a 4-approximation 
of a minimum vertex cover; the algorithm is local and it does not need unique node identifiers.

A more careful analysis shows that $|C| \leq 3\left|C^{*}\right|$, so this is actually a local 3 -approximation algorithm for the vertex cover problem [128]; the bottleneck is a path of length 2 in the set $X$. A repeated application of bicoloured double covers can be used to find a 2-approximation of a minimum vertex cover [7]. See Hańćkowiak et al. [65] for an example of a non-local distributed algorithm that exploits bicoloured double covers.

7.2. Linear programs and vertex covers. In a centralised setting, another 2-approximation algorithm for the vertex cover problem can be obtained by deterministic LP rounding [70]: (i) solve the LP relaxation of the vertex cover problem; and (ii) output the set of nodes $v \in V$ with $x_{v} \geq 1 / 2$. Unlike the algorithm based on a maximal matching, this directly generalises to the problem of finding a minimum-weight vertex cover as well.

To obtain the LP relaxation of a vertex cover instance, we first write the vertex cover instance as a set cover instance. The set cover instance determines an integer program of the form (1) on page 10. The LP relaxation of the vertex cover instance is the corresponding 0/1 covering LP (2).

A local algorithm cannot find an exact solution of a linear program; the problem is inherently non-local. However, local approximation algorithms for packing and covering LPs are known. The first such algorithm was presented by Papadimitriou and Yannakakis [125]. In this simple algorithm, the "capacity" of each constraint in a packing LP is distributed evenly among the adjacent agents; the approximation factor is $\Delta_{I}$. Kuhn and Wattenhofer [101] present improved local approximation algorithms for special cases of packing and covering LPs. Finally, Kuhn et al. [91, 92, 98] present local approximation algorithms for general packing LPs and covering LPs. Among others, they show that $0 / 1$ packing LPs and $0 / 1$ covering LPs admit local approximation schemes in bounded-degree graphs.

Hence we can find a factor $1+\varepsilon$ approximation of the LP relaxation of the minimum vertex cover problem in bounded-degree graphs. Therefore deterministic LP rounding yields a factor $2+\varepsilon$ approximation of a minimum vertex cover in bounded-degree graphs, for any $\varepsilon>0$. Note that the vertex cover problem is a special case of the set cover problem with $\Delta_{K}=2$; the same technique of deterministic LP rounding can be applied to design a local $\left(\Delta_{K}+\varepsilon\right)$-approximation algorithm for the set cover problem in bounded-degree graphs, for an arbitrary $\Delta_{K}$.

It is also possible to use the primal-dual schema $[123,139]$ to design an algorithm that finds an approximation of a minimum vertex cover directly without a rounding step. Moscibroda [117, §6.1] uses this approach to design a $(4+\varepsilon)$-approximation algorithm for the vertex cover problem in bounded-degree graphs.

7.3. Weak colouring. Weak colouring provided the first example of a nontrivial combinatorial problem that admits a local algorithm. Naor and Stockmeyer [121] show that if $\mathcal{G}$ is a bounded-degree graph and every node of $\mathcal{G}$ has an odd degree, then there is a local algorithm that finds a weak 
2-colouring of $\mathcal{G}$. Mayer et al. [115] further show that this is possible without unique identifiers; a port numbering and an orientation is enough.

Let us now show how to find a weak colouring with $\Delta^{O(\Delta)}$ colours, using only a port numbering and an orientation. Each node $v$ is coloured with a vector $\mathbf{x}(v)$ that consists of the following components:

- the out-degree and in-degree of $v$

- $p(u, v)$ for each successor $u$ of $v$, in the order of increasing $p(v, u)$

- $p(u, v)$ for each predecessor $u$ of $v$, in the order of increasing $p(v, u)$.

Example 3. Assume that $v$ has three neighbours, $u_{1}, u_{2}, u_{3}$, with the port numbers $p\left(v, u_{1}\right)=1, p\left(v, u_{2}\right)=2$, and $p\left(v, u_{3}\right)=3$. Assume that the edges are oriented $\left(v, u_{1}\right),\left(u_{2}, v\right)$, and $\left(v, u_{3}\right)$. Then

$$
\mathbf{x}(v)=\left(2,1, p\left(u_{1}, v\right), p\left(u_{3}, v\right), p\left(u_{2}, v\right)\right),
$$

that is, the out-degree, the in-degree, two port numbers for the edges leaving $v$, and one port number for the edges entering $v$. Note that the elements are port numbers of the form $p(\cdot, v)$ but they are ordered by the port numbers $p(v, \cdot)$.

To see that the vectors $\mathbf{x}(v)$ are a weak colouring of the graph, we generalise the argument that we saw in Section 5.4. Let $v$ be an arbitrary node. We need to show that there is a neighbour $u$ of $v$ such that $\mathbf{x}(u) \neq \mathbf{x}(v)$. If the first two components (out-degree and in-degree) are equal in $\mathbf{x}(v)$ and $\mathbf{x}(u)$ for each neighbour $u$ of $v$, we can consider the following two cases.

First, assume that the in-degree of $v$ is $k$ and the out-degree of $v$ is at least $k+1$. Then there are at least $k+1$ successors of $v$, and each successor has $k$ predecessors. By the pigeonhole principle, there are at least two successors of $v$, call them $s$ and $t$, and an index $i$ with the following property: the element $i$ of the vector $\mathbf{x}(s)$ is $p(v, s)$ and the element $i$ of the vector $\mathbf{x}(t)$ is $p(v, t)$. Therefore $\mathbf{x}(s) \neq \mathbf{x}(t)$, and we cannot have $\mathbf{x}(v)=\mathbf{x}(s)=\mathbf{x}(t)$. Put otherwise, $v$ has a different colour from $s$ or $t$ (or both).

Second, assume that the in-degree of $v$ is at least $k+1$ and the out-degree of $v$ is $k$. This case is analogous: we apply the pigeonhole principle to the predecessors of $v$.

Note that this analysis does not go through in a graph with an even degree. We may have in-degrees equal to out-degrees, and therefore we cannot invoke the pigeonhole principle - consider, for example, the 4-regular graph in Figure 5 on page 15 .

So far we have seen how to find a weak colouring with a constant but large number of colours. In the following section, we review techniques that can be used to reduce the number of colours.

7.4. Colour reduction. A local algorithm cannot find a vertex colouring, but it can decrease the number of colours. Given a $k$-colouring for a constant $k$, it is easy to design a local algorithm that finds a $(\Delta+1)$-colouring. In essence, we run a greedy algorithm. The original $k$-colouring partitions the network in $k$ subsets $X_{1}, X_{2}, \ldots, X_{k}$. In the step $i=1,2, \ldots, k$, the nodes in subset $X_{i}$ choose a colour that is not used by any of their neighbours in $X_{1} \cup X_{2} \cup \cdots \cup X_{i-1}$. Each subset $X_{i}$ is an independent set; hence all nodes in $X_{i}$ can make their choices independently in parallel. In the worst case, $\Delta+1$ colours are needed. 
A much more efficient algorithm can be designed by exploiting the numerical values of the original colours. In a cycle, the technique originally presented by Cole and Vishkin [26] allows one to decrease the number of colours from $k$ to $O(\log k)$ in one step. Iterating the procedure, we can turn a $k$-colouring into a 3-colouring with a local algorithm in $O\left(\log ^{*} k\right)$ steps. The textbook by Cormen et al. $[27, \S 30.5]$ has a good illustration of the Cole-Vishkin technique; in essence, a node with a $b$-bit label relabels itself with an $O(\log b)$-bit label $(i, x)$ that identifies the index $i$ and the value $x$ of the first bit in its old label that differs from the next node in the cycle.

The same basic idea can be applied in general bounded-degree graphs [59]. If $k$ is a constant, a $k$-colouring can be turned into a $(\Delta+1)$-colouring with a local algorithm in $O\left(\Delta+\log ^{*} k\right)$ steps [16, 93]; see also Attiya et al.'s [10] algorithm that finds a $(\Delta+1)$-colouring assuming that a so-called $t$-orientation is given. Naor and Stockmeyer [121] show how to turn a weak $k$-colouring into a weak 2-colouring.

Naturally, if we are given a $k$-colouring for a constant $k$, we can also solve a number of other problems with a local algorithm. The symmetry has been broken and, for example, finding a maximal independent set is then easy $[10,13]$. The following algorithm provides a reasonable trade-off between efficiency and simplicity: first apply a colour reduction algorithm, and then find a maximal independent set with a greedy algorithm. However, if $\Delta$ is large, more efficient alternatives exist; see, for example, the techniques used by Schneider and Wattenhofer [130].

7.5. Matchings. As we have seen in Section 6.4, there is no local algorithm for finding a constant-factor approximation of a maximum matching in any family of graphs that contains $n$-cycles. However, positive results are known for bicoloured graphs and for graphs where each node has an odd degree.

We have already seen the algorithm by Hańćkowiak et al. [64] for finding a maximal matching in bicoloured bounded-degree graphs. A maximal matching is a 2-approximation of a maximum matching. It is possible to improve the approximation factor to $1+\varepsilon$ for any $\varepsilon>0$; it is enough to make sure that there is no augmenting path of length $O(1 / \varepsilon)$ [8]. With the help of the bicoloured double cover from Section 7.1, this yields a $(2+\varepsilon)$ approximation of a maximum-size simple 2-matching in general boundeddegree graphs. Maximal matchings can also be used to find approximations of semi-matchings [29].

Bounded-degree graphs where each node has an odd degree admit a local $\Delta$ approximation algorithm for the maximum matching problem; the algorithm proceeds as follows. We can first find a weak 2-colouring $c: V \rightarrow\{1,2\}$ with the algorithm by Naor and Stockmeyer [121]. Let $X \subseteq E$ consist of the edges $\{u, v\} \in E$ with $c(u) \neq c(v)$. Let $\mathcal{H}=(U, X)$ be the subgraph of $\mathcal{G}$ induced by the edges in $X$, that is, $U$ consists of the endpoints of the edges in $X$. Now $\mathcal{H}$ together with $c$ is a bicoloured graph; therefore we can find a maximal matching $M$ in $\mathcal{H}$; this is also a matching in $\mathcal{G}$. Let us now establish the approximation ratio. Let $M^{*}$ be a maximum matching. Let $U \subseteq V$ consist of the nodes matched in $M$ and let $U^{*} \subseteq V$ consists of the nodes matched in $M^{*}$. If $v \in U^{*} \backslash U$, then $v$ is non-isolated in $\mathcal{G}$ and hence it is adjacent to a node $u$ with the opposite colour, by the definition of a weak 
2-colouring. Therefore $\{u, v\}$ is an edge in the subgraph $\mathcal{H}$, and since $M$ is maximal, we have $u \in U$. Furthermore, $u$ is adjacent to at least one node in $U$; therefore $u$ is adjacent to at most $\Delta-1$ nodes in $U^{*} \backslash U$. Summing over all nodes in $U$, we have $\left|U^{*} \backslash U\right| \leq(\Delta-1)|U|$, that is, $\left|U^{*}\right| \leq \Delta|U|$ and $\left|M^{*}\right| \leq \Delta|M|$. The approximation factor can be further improved to $(\Delta+1) / 2$, which is tight [8].

The problem of finding a stable matching is inherently non-local, even in bicoloured graphs. However, it is possible to find an $\varepsilon$-stable matching in a bicoloured bounded-degree graph with a local algorithm [48]. In essence, the local algorithm runs the Gale-Shapley algorithm [54] for a constant number of rounds. The same local algorithm finds a $(2+\varepsilon)$-approximation of a maximum-weight matching in bicoloured bounded-degree graphs.

7.6. Domination. In a bounded-degree graph, the set of all nodes is a factor $\Delta+1$ approximation of a minimum dominating set. If each node has an odd degree, it is possible to find a factor $\Delta$ approximation with a local algorithm, using a port numbering and an orientation: find a weak 2 -colouring $[115,121]$ and output all nodes of colour 1 and all isolated nodes. This set is, by definition, a dominating set: a non-isolated node of colour 2 is adjacent to at least one node of colour 1 . To establish the approximation ratio, assume that $\mathcal{G}=(V, E)$ is connected; otherwise we can apply the result to each connected component. If $|V|=1$, the claim is trivial. Otherwise, let $D^{*}$ be an optimal dominating set in $\mathcal{G}$, let $D_{1}$ consist of the nodes of colour 1 , and let $D_{2}=V \backslash D_{1}$ consist of the nodes of colour 2. Now $D^{*}, D_{1}$, and $D_{2}$ are dominating sets in $\mathcal{G}$. Furthermore, for any dominating set $D$, it holds that $|D| \geq|V| /(\Delta+1)$ because a node in $D$ can only dominate at most $\Delta$ nodes outside $D$. Hence

$$
\left|D_{1}\right|=|V|-\left|D_{2}\right| \leq|V|-\frac{|V|}{\Delta+1}=\frac{\Delta|V|}{\Delta+1} \leq \Delta\left|D^{*}\right|,
$$

that is, $D_{1}$ is a $\Delta$-approximation of a minimum dominating set in $\mathcal{G}$. Moreover, $\left(D_{1}, D_{2}\right)$ is a domatic partition of size 2 if there are no isolated nodes, and a maximum domatic partition has at most $\delta+1$ disjoint dominating sets where $\delta$ is the minimum degree of $\mathcal{G}$. Hence a weak 2-colouring provides a factor $(\delta+1) / 2$ approximation of a maximum domatic partition if there are no isolated nodes; the trivial solution $(V)$ is optimal if there is an isolated node (i.e., if $\delta=0$ ).

It is possible to perform local modifications in a weak 2-colouring so that the number of colour-1 nodes is at most as large as the number of colour-2 nodes [8]. This approach provides a factor $(\Delta+1) / 2$ approximation algorithm for the dominating set problem in graphs of odd degree, and a $\Delta$-approximation in general graphs for an odd $\Delta$.

Czygrinow et al. [30] and Lenzen et al. [104], [103, §13] present a local, constant-factor approximation algorithm for the dominating set problem in planar graphs. The current best known approximation factor is 130 [103, 104].

In Section 7.1 we saw how to use a maximal matching in a bicoloured double cover to find a constant-factor approximation for the vertex cover problem. The same technique provides a 4-approximation of a minimum edge dominating set as well, and with minor modifications the approximation factor can be improved to $4-2 / \Delta[135]$. 
7.7. Trivial algorithms. For some families of graphs, there is a trivial local approximation algorithm for the vertex cover problem. For example, the set of all nodes is a 2-approximation of a minimum vertex cover in regular graphs, and the set of all non-isolated nodes is a 6-approximation of a minimum vertex cover in unit-disk graphs [91, 145].

There is a trivial, local approximation algorithm for the edge cover problem: independently and in parallel, each node $v \in V$ chooses one neighbour $x(v) \in V$ with $\{v, x(v)\} \in E$. The set $C=\{\{v, x(v)\}: v \in V\}$ is a factor 2 approximation of a minimum edge cover. This generalises to the minimum-weight edge cover as well: each node chooses the cheapest edge.

The edge cover problem is a special case of the set cover problem with $\Delta_{V}=2$. The trivial 2-approximation algorithm for the edge cover problem can be applied to approximating set cover as well: each customer $k \in K$ chooses independently and in parallel which agent $v \in V$ covers it. The approximation factor is $\Delta_{V}$.

7.8. Local verification and locally checkable proofs. Korman et al. [8487] and Fraigniaud et al. [51] study the problem of verifying a solution with a local algorithm. We have seen that the problem of finding a spanning tree is inherently non-local, and if we are given a spanning tree in a natural way as a subset of edges, a local algorithm cannot verify whether it really is a spanning tree or not; recall Figure 9 on page 19.

However, it is possible to give a spanning tree together with a proof that can be verified with a local algorithm, so that an invalid input is detected by at least one node (assuming that the communication graph $\mathcal{G}$ is connected). For example, we can orient the spanning tree towards an arbitrary root node. For each node, the proof consists of (i) the identity of the root node, (ii) the distance to the root node in the spanning tree, and (iii) the edge that points towards the root node.

To encode the above proof, we need $O(\log |V|)$ bits per node - we say that the local proof complexity of the spanning tree problem is $O(\log |V|)$. In general, we can classify graph problems according to their local proof complexity: for many classical graph problems, the proof complexity is either $0, \Theta(1), \Theta(\log |V|)$, or poly $(|V|)$ bits per node [60].

7.9. Other problems. Kuhn et al. [97] studies a generalisation of covering LPs, with upper bounds for variables $x_{v}$.

Floréen et al. [42-46] present local algorithms for max-min LP. It is possible to find a factor $\Delta_{I}\left(1-1 / \Delta_{K}\right)+\varepsilon$ approximation of a max-min LP in bounded-degree graphs; this approximation ratio is tight.

A weak 2-colouring also determines a cut with at least $|E| / \Delta$ edges. This gives a partial answer to Elkin's [38] question regarding the distributed approximability of the maximum cut problem.

Positive results for the circuit complexity class $\mathrm{NC}^{0}[6,28,69]$ may also have positive implications in the field of local algorithms; this possible connection calls for further research. 


\section{RANDOMISED LOCAL ALGORITHMS}

In the previous section, we have seen that many graph problems can be solved (at least approximately) by using a deterministic local algorithm. However, the strong negative results of Section 6 make it impossible to solve classical graph problems such as graph colouring, maximal independent sets, maximal matchings, etc., with a deterministic local algorithm. A natural idea is to extend the model by introducing a source of random bits.

8.1. Non-constant guarantees. Randomness is a powerful and classical technique in the design of distributed algorithms, and it is particularly useful in breaking the symmetry $[3,76,111]$. However, in a typical randomised distributed algorithm, either the running time or the performance guarantee depends on the number of nodes.

Luby's [111] algorithm for maximal independent sets is a good example of the former case. When the algorithm terminates, the output is a maximal independent set - however, the expected running time is logarithmic in the number of nodes.

Kuhn's [91, §4.5] algorithm for one-round graph colouring is a good example of the latter case. The algorithm always terminates in one round, and with a high probability, it produces a proper colouring - however, the number of colours is logarithmic in the number of nodes.

As explained in Section 1.1, our focus is on algorithms for which both the running time and the performance guarantees are independent of the number of nodes - that is, we would obtain a finite performance guarantee even in an infinitely large network. In what follows, we examine how much randomness helps in such a limited setting.

8.2. Negative results. There are two key techniques which make randomness a practical tool in the design of centralised algorithms, both familiar from textbooks on randomised algorithms [116, 118]. First, the probability of a "failure" - the event of producing an infeasible output - can be made negligible by adapting the algorithm to the global properties of the input; for example, the number of iterations can depend on the size of the input. Second, it may be possible to detect incorrect output and re-execute the algorithm until the output is correct, turning a Monte Carlo algorithm into a Las Vegas one.

In a strictly local setting, neither of these two techniques can be applied as such. First, the execution of a local algorithm cannot depend on the size of the input. Second, it is not possible to gather the output in a central location for inspection and re-execute the algorithm depending on whether the output is incorrect. Indeed, if a randomised local algorithm has a nonzero probability of failure given some input, then we can simply take several copies of the input to boost the probability that the algorithm makes a failure in at least one copy; see, e.g., Kuhn [91, §4.5].

There are strong negative results on the power of randomness in the local setting. Linial [109] and Naor [120] show that randomness does not help in local algorithms where the objective is to colour a graph, and Kuhn [91, §4.5] extends this to the problem of colour reduction. Naor and Stockmeyer [121] show that randomness does not help in a local algorithm for any locally 
checkable labelling; this includes, among others, the problem of finding a maximal matching or a maximal independent set in a bounded-degree graph.

Nevertheless, there are positive results where a randomised local algorithm provides a probabilistic approximation guarantee. For example, in some cases it is possible to give an upper bound on the expected approximation factor - here the expectation is over the random coin tosses made by the algorithm; nothing is assumed about the input. If we are content with a probabilistic approximation guarantee, it is possible to overcome some of the negative results in Section 6.

Interestingly, Kuhn [91, §2.7.1] shows that probabilistic approximation guarantees do not help with linear programs: if there is a local, randomised algorithm with the expected approximation factor $\alpha$, then there is a local, deterministic $\alpha$-approximation algorithm as well. Therefore all positive examples in this section involve combinatorial problems.

8.3. Matchings and independent sets. As we have seen in Section 6.4, packing problems such as maximum matching and maximum independent set do not admit deterministic, local approximation algorithms, not even in the case of an $n$-cycle with unique node identifiers. With these problems, randomness clearly helps.

Wattenhofer and Wattenhofer [141] present a randomised local approximation algorithm for the maximum-weight matching problem in trees; the expected weight of the matching is within factor 4 of the optimum. Hoepman et al. [72] improve the expected approximation ratio to $2+\varepsilon$.

Nguyen and Onak [122] present a randomised local algorithm for the maximum matching problem in bounded-degree graphs; the approximation ratio is $1+\varepsilon$ with high probability.

Czygrinow et al. [30] present a randomised local algorithm for finding a maximum independent set in a planar graph; the approximation ratio is $1+\varepsilon$ with high probability.

8.4. Maximum cut and maximum satisfiability. Another negative result from Section 6.4 shows that there is no deterministic local approximation algorithm for the maximum cut problem. Again, a randomised local algorithm exists. In this case, we can resort to a very simple algorithm, familiar from introductory courses to randomised algorithms: flip a fair coin for each node to determine its side $[112,116,118]$. The expected size of the cut is $|E| / 2$; hence the expected approximation ratio is 2 . The algorithm is clearly local; no communication is needed.

A similar approach can be applied to the maximum satisfiability (MAXSAT) problem: choose a random assignment $[116,118]$. See, e.g., Ausiello et al. [12, Problem LO1] or Garey and Johnson [55, Problem LO5] for the definition of the problem. However, unlike the maximum cut problem, MAXSAT has a simple, local, deterministic 2-approximation algorithm: First, for each clause, remove all but one of the literals; in essence, we arrive at a MAX-1-SAT instance. Second, satisfy at least half of the clauses by using a local version of Johnson's [79] 2-approximation algorithm.

8.5. LP rounding. Kuhn et al. [91, 97, 98, 101] present a general framework for designing randomised local algorithms for the set cover and set packing 


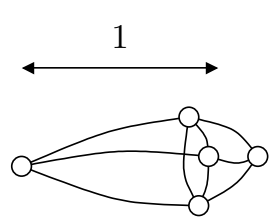

(a)

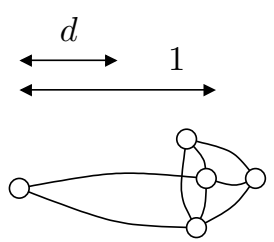

(b)

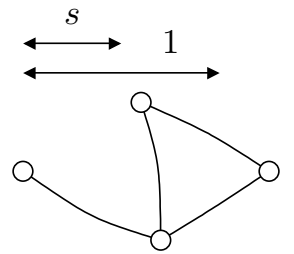

(c)

Figure 13. (a) A unit-disk graph. (b) A quasi unit-disk graph, with $d=1 / 2$. (c) A civilised graph, with $s=1 / 2$. This is also a quasi unit-disk graph, with $d<s$.

problems in bounded-degree graphs. The solution is obtained in three phases: (1) Solve the LP relaxation of the problem approximately with a local algorithm. (2) Apply randomised rounding to find a candidate solution; at this point, the solution is integral but it is not necessarily feasible. (3) Apply a deterministic algorithm to make the solution feasible.

As discussed earlier in Section 7, the LP relaxations of the set cover and set packing problems have local approximation schemes in bounded-degree graphs. Together with these algorithms, the LP rounding scheme yields the expected approximation ratio $O\left(\log \Delta_{V}\right)$ for the set cover problem and $O\left(\Delta_{V}\right)$ for the set packing problem.

In bounded-degree graphs, these results imply the following expected approximation ratios: $O(\log \Delta)$ for vertex covers, $O(\log \Delta)$ for dominating sets, $O(\Delta)$ for maximum independent sets, and $O(1)$ for maximum matchings.

\section{Geometric problems}

Now we turn our attention to geometric graphs. In a geometric graph, each node is embedded in a low-dimensional space, typically in the two-dimensional plane.

9.1. Models. Most research has focused on the case where $\mathcal{G}$ is a unit-disk graph: a pair of nodes is connected by an edge if and only if the Euclidean distance between them is at most 1. See Figure 13a.

Work has also been done on generalisations of unit-disk graphs. A quasi unit-disk graph $[17,102]$ is a graph where the nodes are embedded in the two-dimensional plane, the length of an edge is at most 1, and nodes which are within distance $d$ from each other are always connected by an edge; here $0<d<1$ is a constant. See Figure 13b. A civilised graph (graph drawn in a civilised manner) $[37, \S 8.5]$ is a graph where the nodes are embedded in the two-dimensional plane, the length of an edge is at most 1 , and the distance between any pair of nodes is at least $s$; here $0<s<1$ is a constant. See Figure 13c.

By definition, a civilised graph with parameter $s$ is a quasi unit-disk graph with parameter $d<s$; therefore all positive results for quasi unit-disk graphs apply directly to civilised graphs. Furthermore, a civilised graph is a bounded-degree graph, as can be seen by a simple packing argument. 


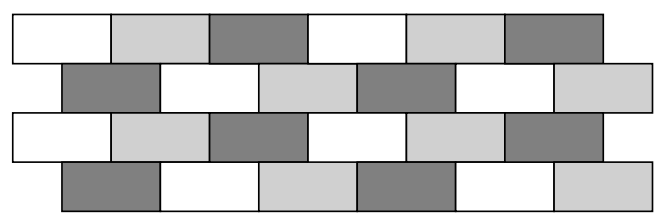

Figure 14. Partitioning the two-dimensional plane into 3coloured rectangles with dimensions $2 \times 1$.

9.2. Partial geometric information. For some problems, it is sufficient to have a local knowledge of the embedding. Kuhn et al. [91, 96] show that packing and covering LPs admit local constant-factor approximation algorithms in unit-disk graphs. It is enough that the distances between the nodes are known so that each node can construct a local coordinate system.

To give another example, Floréen et al. [47, 49] study scheduling problems in a semi-geometric setting in which the coordinates of the nodes are not known, but a small amount of symmetry-breaking information is available.

Most positive results, however, assume that there is a global coordinate system and each node knows its coordinate (so-called location-aware nodes). We review these results in the following.

9.3. Algorithms from simple tilings. A simple approach for designing local algorithms in a geometric setting is to partition the two-dimensional plane into rectangles, and colour the rectangles with a constant number of colours $[67,68,91,96,117,144,146,147]$. Partitioning the two-dimensional plane into rectangles also partitions the network into clusters. If each node knows its coordinates, it knows into which cluster it belongs to.

As a concrete example, we can partition the plane into rectangles of size $2 \times 1$ and colour them with 3 colours so that the distance between a pair of nodes in two different rectangles of the same colour is larger than 1. See Figure 14 for illustration; we use the names white, light, and dark for the colours.

By construction, we know that if nodes $u$ and $v$ are in two different rectangles of the same colour, then there is no edge $\{u, v\}$ in a (quasi) unitdisk graph. Furthermore, a packing argument shows that there is a constant upper bound $D$ on the diameter of a connected component of a cluster. In Awerbuch et al.'s [13] terminology, these coloured rectangles provide a $(3, D)$-decomposition of the network. In Attiya et al.'s [10] terminology, the coloured rectangles provide a $t$-orientation of graph $\mathcal{G}$ for $t=3 D$.

Now it is easy to design a local 3 -approximation algorithm for vertex colouring $[67,91,144,147]$. We handle each connected component in each cluster independently in parallel. A local algorithm finds an optimal vertex colouring within each component; the components have bounded diameter and hence a local algorithm can gather full information about the component. Connected components in white rectangles assign colours $1,4,7, \ldots$, connected components in light rectangles assign colours $2,5,8, \ldots$, and connected components in dark rectangles assign colours $3,6,9, \ldots$ Put together, we obtain a feasible vertex colouring, and the number of colours that we use is within factor 3 of the optimum; see Figure 15. 


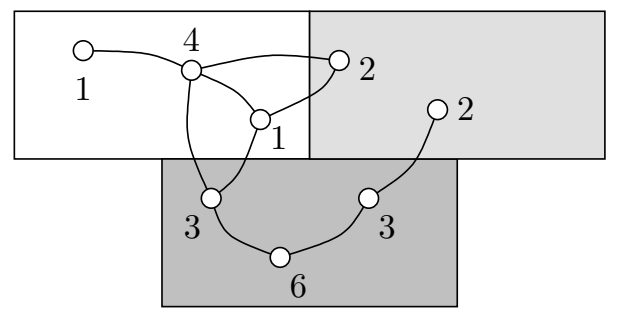

FIgURE 15. Factor 3 approximation for vertex colouring. The edges that cross the boundaries of the tiles can be safely ignored in the algorithm.

A similar idea (with larger 3-coloured rectangles) can be used to design local 3-approximation algorithms for the following problems: edge colouring [67], vertex cover [67, 68, 144], and dominating set [67, 68, 144]. These are examples of local algorithms that unscrupulously exploit the assumption that local computation is free; nevertheless, Hunt et al. [74] show how to solve the subproblem of finding a minimum-size dominating set or vertex cover within a rectangle in polynomial time.

Another algorithm design technique that employs the same 3-coloured tiling is the sequential greedy strategy. Consider, for example, the task of finding a maximal independent set. We can proceed in three phases as follows $[10$, 13, 67]. First, each white rectangle finds a maximal independent set with a greedy algorithm. Then each light rectangle extends the independent set greedily, taking into account the output in neighbouring white rectangles. Finally, each dark rectangle extends the independent set greedily, taking into account neighbouring white and light rectangles. The same technique can be applied to find a maximal matching $[67,146]$ and a vertex $(\Delta+1)$ colouring $[10,13,67]$. A local algorithm for maximal matching then gives a 2-approximation of a minimum vertex cover as well.

Finally, it is possible to find a factor 4 approximation of a maximum independent set by using a similar 3-coloured tiling [67, 144]. First, each cluster finds a maximum-size independent set in parallel. This may cause conflicts. The conflicts are then resolved; first those that involve white rectangles and then those that involve light rectangles. At each conflict resolution we lose at most one half of the nodes; hence the remaining nodes provide a factor 4 approximation.

9.4. Other algorithms. Wiese and Kranakis [144, 146, 148, 149] present local approximation schemes for dominating sets, connected dominating sets, vertex covers, maximum matchings, and maximum independent sets in unit-disk graphs.

Czyzowicz et al. [31] present a 5-approximation algorithm for the dominating set problem and a 7.46-approximation algorithm for the connected dominating set problem. Wiese and Kranakis $[144,145]$ study local approximation algorithms with local horizon $r \leq 2$ for dominating sets, connected dominating sets, vertex covers, and independent sets in unit-disk graphs. Kuhn and Moscibroda [94] present a local approximation algorithm for the capacitated dominating set problem in unit-disk graphs; this is a variant of 
the dominating set problem in which each node has a limited capacity that determines how many neighbours it can dominate.

Šparl and Žerovnik [132] present a 4/3-approximation algorithm for multicolouring hexagonal graphs. Kaski et al. [82] present a local approximation scheme for link scheduling (a variant of the graph colouring problem) in geometric graphs.

9.5. Planar subgraphs and geographic routing. In geographic routing $[56,153]$, it is of interest to construct a connected planar subgraph $\mathcal{H}=\left(V, E^{\prime}\right)$ of a unit-disk graph $\mathcal{G}=(V, E)$, with the original set of nodes $V$ but a smaller set of edges $E^{\prime} \subset E$.

There are local algorithms for constructing planar subgraphs. For example, Gabriel graphs [53] and relative neighbourhood graphs [78, 137] can be constructed with simple local rules.

Once we have constructed a planar subgraph of a unit-disk graph, it is possible to route messages with local geometric rules, assuming that we know the coordinates of the target node $[20,89]$.

9.6. Spanners. In applications such as topology control, merely having a connected planar subgraph $\mathcal{H}$ is not enough. Among others, it is desirable that $\mathcal{H}$ is a geometric $t$-spanner. In a $t$-spanner, for any pair $u, v$ of nodes in $\mathcal{G}$, the shortest path between $u$ and $v$ in $\mathcal{H}$ is at most $t$ times as long as the shortest path between $u$ and $v$ in $\mathcal{G}$; here the length of a path is the sum of the Euclidean lengths of the edges. The constant $t$ is the stretch factor of the spanner.

Gabriel graphs and relative neighbourhood graphs are not $t$-spanners for any constant $t$. Yao graphs [152] and $\theta$-graphs [83] provide classical examples of spanners that can be constructed with a simple local algorithm. However, these constructions lack some desirable properties; in particular, they do not have a constant upper bound on the node degree.

Examples of more recent work include the following. Wang and Li [140] present a local algorithm for constructing a planar, bounded-degree spanner in unit-disk graphs. The local algorithms by Li et al. [108] and Kanj et al. [81] further guarantee that the total edge length of the spanner is at most a constant factor larger than the total edge length of a minimum spanning tree. Chávez et al. [23] generalise the results by Li et al. [108] to quasi unit-disk graphs. Wattenhofer and Zollinger [142] present a local algorithm that can be applied in arbitrary weighted graphs, not only in geometric graphs.

9.7. Coloured subgraphs. Local algorithms have also been presented for constructing coloured subgraphs. Urrutia [138] presents a local algorithm that constructs a connected, planar, edge-coloured subgraph of a unit-disk graph. Wiese and Kranakis $[144,147]$ present a local algorithm that constructs a connected, planar, vertex-coloured subgraph of a unit-disk graph. Czyzowicz et al. [32] present a local algorithm for colouring the nodes in an arbitrary planar subgraph of a unit-disk graph. Czyzowicz et al. [33] present a local algorithm for colouring the edges in certain subgraphs of unit-disk graphs. 


\section{Open PROBLEMS}

We conclude this survey with some open problems related to deterministic local algorithms. We recall that in this work a local algorithm refers to a constant-time algorithm.

Problem 1. Is there a local approximation scheme for general packing LPs or covering LPs in bounded-degree graphs?

The local approximation scheme by Kuhn et al. [98] assumes not only a degree bound but also an upper bound for the ratio of largest coefficient to smallest coefficient in the LP. Techniques by Luby and Nisan [113] and Bartal et al. [18] can be applied to avoid the dependency on coefficients, but this comes at the cost of adding a dependency on the size of the input [99].

Problem 2. Is there a combinatorial packing problem that admits a nontrivial, deterministic, local approximation algorithm?

Finding a simple 2-matching is a packing problem, but it is a slightly contrived example. It would be interesting to see other, more natural examples of packing problems that can be solved locally, without any auxiliary information.

A partial answer is provided by the local approximation algorithm for the maximum matching problem, based on the weak 2-colouring algorithm by Naor and Stockmeyer [121]. However, this can be applied only in a graph where every node has an odd degree, a rather stringent assumption.

Problem 3. Is there a problem that (i) can be solved with a local algorithm that exploits the numerical values of the identifiers, and (ii) cannot be solved with an order-invariant local algorithm that merely compares the identifiers?

Naor and Stockmeyer [121] show that order-invariant local algorithms are sufficient for locally checkable labellings: if there is a local algorithm for a locally checkable labelling problem, then there is an order-invariant algorithm as well. Hence we need to seek for an example outside locally checkable labellings.

If we assume that the set of node identifiers is $\{1,2, \ldots,|V|\}$, then we can find some examples of problems that admit a local algorithm and do not admit an order-invariant local algorithm. For example, in this case leader election is trivial with a local algorithm (the node number 1 is the leader) but there is no order-invariant local algorithm for the task. However, this example is no longer valid if the unique node identifiers are an arbitrary subset of, say, $\{1,2, \ldots, 2|V|\}$.

Problem 4. How does the local horizon depend on $\Delta$ ?

Typically, the running time of a local algorithm depends on the maximum degree $\Delta$. However, we do not yet understand thoroughly how the local horizon depends on $\Delta$ : in many cases, the fastest known algorithms have running times that are polynomial in $\Delta[9,16,48,64,93,135]$ while the best known lower bounds are typically polylogarithmic in $\Delta[95,100]$. 


\section{ACKNOWLEDGEMENTS}

This is the author's version of the work; the definitive version will be published in ACM Computing Surveys [136]. A preliminary version of this survey was published as a part of my $\mathrm{PhD}$ thesis $[134, \S 2]$. Updates to this work will be posted online at http://www.cs.helsinki.fi/local-survey/

I am grateful to Patrik Floréen, Keijo Heljanko, Juho Hirvonen, Petteri Kaski, Evangelos Kranakis, Christoph Lenzen, Valentin Polishchuk, Joel Rybicki, Christian Scheideler, Roger Wattenhofer, and anonymous reviewers for their helpful comments and suggestions.

This work was supported in part by the Academy of Finland, Grants 116547, 132380, and 252018, by Helsinki Graduate School in Computer Science and Engineering (Hecse), by the Foundation of Nokia Corporation, by the Finnish Cultural Foundation, and by Research Funds of the University of Helsinki.

\section{REFERENCES}

[1] Scott Aaronson, Greg Kuperberg, and Christopher Granade. Complexity zoo. http: //qwiki.stanford.edu/index.php/Complexity_Zoo, August 2011.

[2] Robert B. Allan and Renu Laskar. On domination and independent domination numbers of a graph. Discrete Mathematics, 23(2):73-76, 1978.

[3] Noga Alon, László Babai, and Alon Itai. A fast and simple randomized parallel algorithm for the maximal independent set problem. Journal of Algorithms, 7(4):567583, 1986.

[4] Alon Amit, Nathan Linial, Jiří Matoušek, and Eyal Rozenman. Random lifts of graphs. In Proc. 12th Annual ACM-SIAM Symposium on Discrete Algorithms (SODA, Washington, DC, USA, January 2001), pages 883-894. Society for Industrial and Applied Mathematics, Philadelphia, PA, USA, 2001.

[5] Dana Angluin. Local and global properties in networks of processors. In Proc. 12th Annual ACM Symposium on Theory of Computing (STOC, Los Angeles, CA, USA, April 1980), pages 82-93. ACM Press, New York, NY, USA, 1980.

[6] Benny Applebaum, Yuval Ishai, and Eyal Kushilevitz. Cryptography in $\mathrm{NC}^{0}$. SIAM Journal on Computing, 36(4):845-888, 2006.

[7] Matti Åstrand, Patrik Floréen, Valentin Polishchuk, Joel Rybicki, Jukka Suomela, and Jara Uitto. A local 2-approximation algorithm for the vertex cover problem. In Proc. 23rd International Symposium on Distributed Computing (DISC, Elche, Spain, September 2009), volume 5805 of Lecture Notes in Computer Science, pages 191-205. Springer, Berlin, Germany, 2009.

[8] Matti Åstrand, Valentin Polishchuk, Joel Rybicki, Jukka Suomela, and Jara Uitto. Local algorithms in (weakly) coloured graphs, 2010. Manuscript, arXiv:1002.0125 [cs.DC].

[9] Matti Åstrand and Jukka Suomela. Fast distributed approximation algorithms for vertex cover and set cover in anonymous networks. In Proc. 22nd Annual ACM Symposium on Parallelism in Algorithms and Architectures (SPAA, Santorini, Greece, June 2010), pages 294-302. ACM Press, New York, NY, USA, 2010.

[10] Hagit Attiya, Hadas Shachnai, and Tami Tamir. Local labeling and resource allocation using preprocessing. SIAM Journal on Computing, 28(4):1397-1413, 1999.

[11] Hagit Attiya, Marc Snir, and Manfred K. Warmuth. Computing on an anonymous ring. Journal of the ACM, 35(4):845-875, 1988.

[12] Giorgio Ausiello, Pierluigi Crescenzi, Giorgio Gambosi, Viggo Kann, Alberto Marchetti-Spaccamela, and Marco Protasi. Complexity and Approximation: Combinatorial Optimization Problems and Their Approximability Properties. Springer, Berlin, Germany, 2003. 
[13] Baruch Awerbuch, Andrew V. Goldberg, Michael Luby, and Serge A. Plotkin. Network decomposition and locality in distributed computation. In Proc. 30th Annual Symposium on Foundations of Computer Science (FOCS, Research Triangle Park, NC, USA, October-November 1989), pages 364-369. IEEE, Piscataway, NJ, USA, 1989.

[14] Baruch Awerbuch and Michael Sipser. Dynamic networks are as fast as static networks. In Proc. 29th Annual Symposium on Foundations of Computer Science (FOCS, White Plains, NY, USA, October 1988), pages 206-219. IEEE, Piscataway, NJ, USA, 1988.

[15] Baruch Awerbuch and George Varghese. Distributed program checking: a paradigm for building self-stabilizing distributed protocols. In Proc. 32nd Annual Symposium on Foundations of Computer Science (FOCS, San Juan, Puerto Rico, October 1991), pages 258-267. IEEE, Piscataway, NJ, USA, 1991.

[16] Leonid Barenboim and Michael Elkin. Distributed $(\Delta+1)$-coloring in linear (in $\Delta$ ) time. In Proc. 41st Annual ACM Symposium on Theory of Computing (STOC, Bethesda, MD, USA, May-June 2009), pages 111-120. ACM Press, New York, NY, USA, 2009.

[17] Lali Barrière, Pierre Fraigniaud, Lata Narayanan, and Jaroslav Opatrny. Robust position-based routing in wireless ad-hoc networks with irregular transmission ranges. Wireless Communications and Mobile Computing Journal, 3(2):141-153, 2003.

[18] Yair Bartal, John W. Byers, and Danny Raz. Global optimization using local information with applications to flow control. In Proc. 38th Annual Symposium on Foundations of Computer Science (FOCS, Miami Beach, FL, USA, October 1997), pages 303-312. IEEE Computer Society Press, Los Alamitos, CA, USA, 1997.

[19] Paolo Boldi and Sebastiano Vigna. An effective characterization of computability in anonymous networks. In Proc. 15th International Symposium on Distributed Computing (DISC, Lisbon, Portugal, October 2001), volume 2180 of Lecture Notes in Computer Science, pages 33-47. Springer, Berlin, Germany, 2001.

[20] Prosenjit Bose, Pat Morin, Ivan Stojmenović, and Jorge Urrutia. Routing with guaranteed delivery in ad hoc wireless networks. Wireless Networks, 7(6):609-616, 2001.

[21] Anne Bottreau and Yves Métivier. The Kronecker product and local computations in graphs. In Proc. 21st International Colloquium on Trees in Algebra and Programming (CAAP, Linköping, Sweden, April 1996), volume 1059 of Lecture Notes in Computer Science, pages 2-16. Springer, Berlin, Germany, 1996.

[22] Mihaela Cardei, David MacCallum, Maggie Xiaoyan Cheng, Manki Min, Xiaohua Jia, Deying Li, and Ding-Zhu Du. Wireless sensor networks with energy efficient organization. Journal of Interconnection Networks, 3(3-4):213-229, 2002.

[23] Edgar Chávez, Stefan Dobrev, Evangelos Kranakis, Jaroslav Opatrny, Ladislav Stacho, and Jorge Urrutia. Local construction of planar spanners in unit disk graphs with irregular transmission ranges. In Proc. 7th Latin American Theoretical Informatics Symposium (LATIN, Valdivia, Chile, March 2006), volume 3887 of Lecture Notes in Computer Science, pages 286-297. Springer, Berlin, Germany, 2006.

[24] Miroslav Chlebík and Janka Chlebíková. Approximation hardness of edge dominating set problems. Journal of Combinatorial Optimization, 11(3):279-290, 2006.

[25] Ernest J. Cockayne and Stephen T. Hedetniemi. Optimal domination in graphs. IEEE Transactions on Circuits and Systems, 22(11):855-857, 1975.

[26] Richard Cole and Uzi Vishkin. Deterministic coin tossing with applications to optimal parallel list ranking. Information and Control, 70(1):32-53, 1986.

[27] Thomas H. Cormen, Charles E. Leiserson, and Ronald L. Rivest. Introduction to Algorithms. The MIT Press, Cambridge, MA, USA, 1990.

[28] Mary Cryan and Peter Bro Miltersen. On pseudorandom generators in $\mathrm{NC}^{0}$. In Proc. 26th International Symposium on Mathematical Foundations of Computer Science (MFCS, Mariánské Lázně, Czech Republic, August 2001), volume 2136 of Lecture Notes in Computer Science, pages 272-284. Springer, Berlin, Germany, 2001.

[29] Andrzej Czygrinow, Michal Hanćkowiak, Krzysztof Krzywdziński, Edyta Szymańska, and Wojciech Wawrzyniak. Brief announcement: Distributed approximations for 
the semi-matching problem. In Proc. 25th International Symposium on Distributed Computing (DISC, Rome, Italy, September 2011), volume 6950 of Lecture Notes in Computer Science, pages 200-201. Springer, Berlin, Germany, 2011.

[30] Andrzej Czygrinow, Michał Hańćkowiak, and Wojciech Wawrzyniak. Fast distributed approximations in planar graphs. In Proc. 22nd International Symposium on Distributed Computing (DISC, Arcachon, France, September 2008), volume 5218 of Lecture Notes in Computer Science, pages 78-92. Springer, Berlin, Germany, 2008.

[31] Jurek Czyzowicz, Stefan Dobrev, Thomas Fevens, Hernán González-Aguilar, Evangelos Kranakis, Jaroslav Opatrny, and Jorge Urrutia. Local algorithms for dominating and connected dominating sets of unit disk graphs with location aware nodes. In Proc. 8th Latin American Theoretical Informatics Symposium (LATIN, Búzios, Brazil, April 2008), volume 4957 of Lecture Notes in Computer Science, pages 158-169. Springer, Berlin, Germany, 2008.

[32] Jurek Czyzowicz, Stefan Dobrev, Hernán González-Aguilar, Rastislav Královič, Evangelos Kranakis, Jaroslav Opatrny, Ladislav Stacho, and Jorge Urrutia. Local 7-coloring for planar subgraphs of unit disk graphs. Theoretical Computer Science, 412(18):1696-1704, 2011.

[33] Jurek Czyzowicz, Stefan Dobrev, Evangelos Kranakis, Jaroslav Opatrny, and Jorge Urrutia. Local edge colouring of Yao-like subgraphs of unit disk graphs. Theoretical Computer Science, 410(14):1388-1400, 2009.

[34] Reinhard Diestel. Graph Theory. Springer, Berlin, Germany, 3rd edition, 2005.

[35] Edsger W. Dijkstra. Self-stabilizing systems in spite of distributed control. Communications of the ACM, 17(11):643-644, 1974.

[36] Shlomi Dolev. Self-Stabilization. The MIT Press, Cambridge, MA, USA, 2000.

[37] Peter G. Doyle and J. Laurie Snell. Random Walks and Electric Networks. Number 22 in The Carus Mathematical Monographs. The Mathematical Association of America, Washington, DC, USA, 1984.

[38] Michael Elkin. Distributed approximation: a survey. ACM SIGACT News, 35(4):40$57,2004$.

[39] David Eppstein, Zvi Galil, and Giuseppe F. Italiano. Dynamic graph algorithms. In Mikhail J. Atallah, editor, Algorithms and Theory of Computation Handbook, chapter 8. CRC Press, Boca Raton, FL, USA, 1999.

[40] Uriel Feige, Magnús M. Halldórsson, Guy Kortsarz, and Aravind Srinivasan. Approximating the domatic number. SIAM Journal on Computing, 32(1):172-195, 2002.

[41] Faith Fich and Eric Ruppert. Hundreds of impossibility results for distributed computing. Distributed Computing, 16(2-3):121-163, 2003.

[42] Patrik Floréen, Marja Hassinen, Joel Kaasinen, Petteri Kaski, Topi Musto, and Jukka Suomela. Local approximability of max-min and min-max linear programs. Theory of Computing Systems, 49(4):672-697, 2011.

[43] Patrik Floréen, Marja Hassinen, Petteri Kaski, and Jukka Suomela. Local approximation algorithms for a class of 0/1 max-min linear programs, 2008. Manuscript, arXiv:0806.0282 [cs.DC].

[44] Patrik Floréen, Marja Hassinen, Petteri Kaski, and Jukka Suomela. Tight local approximation results for max-min linear programs. In Proc. 4th International Workshop on Algorithmic Aspects of Wireless Sensor Networks (Algosensors, Reykjavik, Iceland, July 2008), volume 5389 of Lecture Notes in Computer Science, pages 2-17. Springer, Berlin, Germany, 2008.

[45] Patrik Floréen, Joel Kaasinen, Petteri Kaski, and Jukka Suomela. An optimal local approximation algorithm for max-min linear programs. In Proc. 21st Annual ACM Symposium on Parallelism in Algorithms and Architectures (SPAA, Calgary, Canada, August 2009), pages 260-269. ACM Press, New York, NY, USA, 2009.

[46] Patrik Floréen, Petteri Kaski, Topi Musto, and Jukka Suomela. Approximating max-min linear programs with local algorithms. In Proc. 22nd IEEE International Parallel and Distributed Processing Symposium (IPDPS, Miami, FL, USA, April 2008). IEEE, Piscataway, NJ, USA, 2008. 
[47] Patrik Floréen, Petteri Kaski, Topi Musto, and Jukka Suomela. Local approximation algorithms for scheduling problems in sensor networks. In Proc. 3rd International Workshop on Algorithmic Aspects of Wireless Sensor Networks (Algosensors, Wroctaw, Poland, July 2007), volume 4837 of Lecture Notes in Computer Science, pages 99-113. Springer, Berlin, Germany, 2008.

[48] Patrik Floréen, Petteri Kaski, Valentin Polishchuk, and Jukka Suomela. Almost stable matchings by truncating the Gale-Shapley algorithm. Algorithmica, 58(1):102-118, 2010.

[49] Patrik Floréen, Petteri Kaski, and Jukka Suomela. A distributed approximation scheme for sleep scheduling in sensor networks. In Proc. 4th Annual IEEE Communications Society Conference on Sensor, Mesh and Ad Hoc Communications and Networks (SECON, San Diego, CA, USA, June 2007), pages 152-161. IEEE, Piscataway, NJ, USA, 2007.

[50] Pierre Fraigniaud, Cyril Gavoille, David Ilcinkas, and Andrzej Pelc. Distributed computing with advice: Information sensitivity of graph coloring. In Proc. 34th International Colloquium on Automata, Languages and Programming (ICALP, Wroctaw, Poland, July 2007), volume 4596 of Lecture Notes in Computer Science, pages 231-242. Springer, Berlin, Germany, 2007.

[51] Pierre Fraigniaud, Amos Korman, and David Peleg. Local distributed decision. In Proc. 52nd Annual IEEE Symposium on Foundations of Computer Science (FOCS, Palm Springs, CA, USA, October 2011). IEEE Computer Society Press, Los Alamitos, CA, USA, 2011. To appear.

[52] Toshihiro Fujito and Hiroshi Nagamochi. A 2-approximation algorithm for the minimum weight edge dominating set problem. Discrete Applied Mathematics, 118(3):199-207, 2002.

[53] K. Ruben Gabriel and Robert R. Sokal. A new statistical approach to geographic variation analysis. Systematic Zoology, 18(3):259-278, 1969.

[54] David Gale and Lloyd S. Shapley. College admissions and the stability of marriage. The American Mathematical Monthly, 69(1):9-15, 1962.

[55] Michael R. Garey and David S. Johnson. Computers and Intractability: A Guide to the Theory of NP-Completeness. W. H. Freeman and Company, New York, NY, USA, 1979.

[56] Laszek Gạsieniec, Chang Su, and Prudence Wong. Routing in geometric networks. In Ming-Yang Kao, editor, Encyclopedia of Algorithms. Springer, New York, NY, USA, 2008.

[57] Phillip B. Gibbons. Fun with networks: social, sensor, and shape shifting. Invited talk, 22nd International Symposium on Distributed Computing (DISC, Arcachon, France), September 2008.

[58] Chris Godsil and Gordon Royle. Algebraic Graph Theory, volume 207 of Graduate Texts in Mathematics. Springer, New York, NY, USA, 2004.

[59] Andrew V. Goldberg, Serge A. Plotkin, and Gregory E. Shannon. Parallel symmetrybreaking in sparse graphs. SIAM Journal on Discrete Mathematics, 1(4):434-446, 1988.

[60] Mika Göös and Jukka Suomela. Locally checkable proofs. In Proc. 30th Annual ACM Symposium on Principles of Distributed Computing (PODC, San Jose, CA, USA, June 2011), pages 159-168. ACM Press, New York, NY, USA, 2011.

[61] Ronald L. Graham, Bruce L. Rothschild, and Joel H. Spencer. Ramsey Theory. John Wiley \& Sons, New York, NY, USA, 1980.

[62] Dan Gusfield and Robert W. Irving. The Stable Marriage Problem: Structure and Algorithms. Foundations of Computing. The MIT Press, Cambridge, MA, USA, 1989.

[63] Bruce Hajek and Galen Sasaki. Link scheduling in polynomial time. IEEE Transactions on Information Theory, 34(5):910-917, 1988.

[64] Michał Hańćkowiak, Michał Karoński, and Alessandro Panconesi. On the distributed complexity of computing maximal matchings. In Proc. 9th Annual ACM-SIAM Symposium on Discrete Algorithms (SODA, San Francisco, CA, USA, January 1998), pages 219-225. Society for Industrial and Applied Mathematics, Philadelphia, 
PA, USA, 1998.

[65] Michał Hańćkowiak, Michał Karoński, and Alessandro Panconesi. On the distributed complexity of computing maximal matchings. SIAM Journal on Discrete Mathematics, 15(1):41-57, 2001.

[66] Nicholas J. A. Harvey, Richard E. Ladner, László Lovász, and Tami Tamir. Semimatchings for bipartite graphs and load balancing. Journal of Algorithms, 59(1):53-78, 2006.

[67] Marja Hassinen, Joel Kaasinen, Evangelos Kranakis, Valentin Polishchuk, Jukka Suomela, and Andreas Wiese. Analysing local algorithms in location-aware quasiunit-disk graphs. Discrete Applied Mathematics, 159(15):1566-1580, 2011.

[68] Marja Hassinen, Valentin Polishchuk, and Jukka Suomela. Local 3-approximation algorithms for weighted dominating set and vertex cover in quasi unit-disk graphs. In Proc. 2nd International Workshop on Localized Algorithms and Protocols for Wireless Sensor Networks (LOCALGOS, Santorini Island, Greece, June 2008), pages V.9-V.12. 2008.

[69] Johan Håstad. One-way permutations in $\mathrm{NC}^{0}$. Information Processing Letters, 26(3):153-155, 1987.

[70] Dorit S. Hochbaum. Approximation algorithms for the set covering and vertex cover problems. SIAM Journal on Computing, 11(3):555-556, 1982.

[71] John G. Hocking and Gail S. Young. Topology. Addison-Wesley, Reading, MA, USA, 1961.

[72] Jaap-Henk Hoepman, Shay Kutten, and Zvi Lotker. Efficient distributed weighted matchings on trees. In Proc. 13th International Colloquium on Structural Information and Communication Complexity (SIROCCO, Chester, UK, July 2006), volume 4056 of Lecture Notes in Computer Science, pages 115-129. Springer, Berlin, Germany, 2006.

[73] Shlomo Hoory. On Graphs of High Girth. PhD thesis, Hebrew University, Jerusalem, March 2002.

[74] Harry B. Hunt III, Madhav V. Marathe, Venkatesh Radhakrishnan, S. S. Ravi, Daniel J. Rosenkrantz, and Richard E. Stearns. NC-approximation schemes for NP- and PSPACE-hard problems for geometric graphs. Journal of Algorithms, 26(2):238-274, 1998.

[75] Wilfried Imrich and Tomaž Pisanski. Multiple Kronecker covering graphs. European Journal of Combinatorics, 29(5):1116-1122, 2008.

[76] Amos Israeli and Alon Itai. A fast and simple randomized parallel algorithm for maximal matching. Information Processing Letters, 22(2):77-80, 1986.

[77] Kamal Jain, Jitendra Padhye, Venkata N. Padmanabhan, and Lili Qiu. Impact of interference on multi-hop wireless network performance. Wireless Networks, 11(4):471-487, 2005.

[78] Jerzy W. Jaromczyk and Godfried T. Toussaint. Relative neighborhood graphs and their relatives. Proceedings of the IEEE, 80(9):1502-1517, 1992.

[79] David S. Johnson. Approximation algorithms for combinatorial problems. Journal of Computer and System Sciences, 9:256-278, 1974.

[80] Ralph E. Johnson and Fred B. Schneider. Symmetry and similarity in distributed systems. In Proc. 4th Annual ACM Symposium on Principles of Distributed Computing (PODC, Minaki, Ontario, Canada, August 1985), pages 13-22. ACM Press, New York, NY, USA, 1985.

[81] Iyad A. Kanj, Ljubomir Perković, and Ge Xia. Computing lightweight spanners locally. In Proc. 22nd International Symposium on Distributed Computing (DISC, Arcachon, France, September 2008), volume 5218 of Lecture Notes in Computer Science, pages 365-378. Springer, Berlin, Germany, 2008.

[82] Petteri Kaski, Aleksi Penttinen, and Jukka Suomela. Coordinating concurrent transmissions: A constant-factor approximation of maximum-weight independent set in local conflict graphs. Ad Hoc \& Sensor Wireless Networks: An International Journal, 6(3-4):239-263, 2008.

[83] J. Mark Keil and Carl A. Gutwin. Classes of graphs which approximate the complete Euclidean graph. Discrete E Computational Geometry, 7(1):13-28, 1992. 
[84] Amos Korman and Shay Kutten. On distributed verification. In Proc. 8th International Conference on Distributed Computing and Networking (ICDCN, Guwahati, India, December 2006), volume 4308 of Lecture Notes in Computer Science, pages 100-114. Springer, Berlin, Germany, 2006.

[85] Amos Korman and Shay Kutten. Distributed verification of minimum spanning trees. Distributed Computing, 20(4):253-266, 2007.

[86] Amos Korman, Shay Kutten, and David Peleg. Proof labeling schemes. In Proc. 24th Annual ACM Symposium on Principles of Distributed Computing (PODC, Las Vegas, NV, USA, July 2005), pages 9-18. ACM Press, New York, NY, USA, 2005.

[87] Amos Korman, David Peleg, and Yoav Rodeh. Constructing labeling schemes through universal matrices. Algorithmica, 57(4):641-652, 2010.

[88] Bernhard Korte and Jens Vygen. Combinatorial Optimization: Theory and Algorithms. Springer, Berlin, Germany, 3rd edition, 2006.

[89] Evangelos Kranakis, Harvinder Singh, and Jorge Urrutia. Compass routing on geometric networks. In Proc. 11th Canadian Conference on Computational Geometry (CCCG, Vancouver, BC, Canada, August 1999). 1999.

[90] Bhaskar Krishnamachari. Networking Wireless Sensors. Cambridge University Press, Cambridge, UK, 2005.

[91] Fabian Kuhn. The Price of Locality: Exploring the Complexity of Distributed Coordination Primitives. PhD thesis, ETH Zurich, 2005.

[92] Fabian Kuhn. Local approximation of covering and packing problems. In Ming-Yang Kao, editor, Encyclopedia of Algorithms. Springer, New York, NY, USA, 2008.

[93] Fabian Kuhn. Weak graph colorings: Distributed algorithms and applications. In Proc. 21st Annual ACM Symposium on Parallelism in Algorithms and Architectures (SPAA, Calgary, Canada, August 2009), pages 138-144. ACM Press, New York, NY, USA, 2009.

[94] Fabian Kuhn and Thomas Moscibroda. Distributed approximation of capacitated dominating sets. In Proc. 19th Annual ACM Symposium on Parallelism in Algorithms and Architectures (SPAA, San Diego, CA, USA, June 2007), pages 161-170. ACM Press, New York, NY, USA, 2007.

[95] Fabian Kuhn, Thomas Moscibroda, and Roger Wattenhofer. What cannot be computed locally! In Proc. 23rd Annual ACM Symposium on Principles of Distributed Computing (PODC, St. John's, Newfoundland, Canada, July 2004), pages 300-309. ACM Press, New York, NY, USA, 2004.

[96] Fabian Kuhn, Thomas Moscibroda, and Roger Wattenhofer. On the locality of bounded growth. In Proc. 24th Annual ACM Symposium on Principles of Distributed Computing (PODC, Las Vegas, NV, USA, July 2005), pages 60-68. ACM Press, New York, NY, USA, 2005.

[97] Fabian Kuhn, Thomas Moscibroda, and Roger Wattenhofer. Fault-tolerant clustering in ad hoc and sensor networks. In Proc. 26th IEEE International Conference on Distributed Computing Systems (ICDCS, Lisboa, Portugal, July 2006). IEEE Computer Society Press, Los Alamitos, CA, USA, 2006.

[98] Fabian Kuhn, Thomas Moscibroda, and Roger Wattenhofer. The price of being near-sighted. In Proc. 17th Annual ACM-SIAM Symposium on Discrete Algorithms (SODA, Miami, FL, USA, January 2006), pages 980-989. ACM Press, New York, NY, USA, 2006.

[99] Fabian Kuhn, Thomas Moscibroda, and Roger Wattenhofer. The price of being nearsighted. Technical Report 229, ETH Zurich, Computer Engineering and Networks Laboratory, January 2006.

[100] Fabian Kuhn, Thomas Moscibroda, and Roger Wattenhofer. Local computation: Lower and upper bounds, 2010. Manuscript, arXiv:1011.5470 [cs.DC].

[101] Fabian Kuhn and Roger Wattenhofer. Constant-time distributed dominating set approximation. Distributed Computing, 17(4):303-310, 2005.

[102] Fabian Kuhn, Roger Wattenhofer, and Aaron Zollinger. Ad hoc networks beyond unit disk graphs. Wireless Networks, 14(5):715-729, 2008.

[103] Christoph Lenzen. Synchronization and Symmetry Breaking in Distributed Systems. PhD thesis, ETH Zurich, January 2011. 
[104] Christoph Lenzen, Yvonne Anne Oswald, and Roger Wattenhofer. What can be approximated locally? TIK Report 331, ETH Zurich, Computer Engineering and Networks Laboratory, November 2010.

[105] Christoph Lenzen, Jukka Suomela, and Roger Wattenhofer. Local algorithms: Selfstabilization on speed. In Proc. 11th International Symposium on Stabilization, Safety, and Security of Distributed Systems (SSS, Lyon, France, November 2009), volume 5873 of Lecture Notes in Computer Science, pages 17-34. Springer, Berlin, Germany, 2009.

[106] Christoph Lenzen and Roger Wattenhofer. Leveraging Linial's locality limit. In Proc. 22nd International Symposium on Distributed Computing (DISC, Arcachon, France, September 2008), volume 5218 of Lecture Notes in Computer Science, pages 394-407. Springer, Berlin, Germany, 2008.

[107] Christoph Lenzen and Roger Wattenhofer. Minimum dominating set approximation in graphs of bounded arboricity. In Proc. 24th International Symposium on Distributed Computing (DISC, Cambridge, MA, USA, September 2010), volume 6343 of Lecture Notes in Computer Science, pages 510-524. Springer, Berlin, Germany, 2010.

[108] Xiang-Yang Li, Yu Wang, and Wen-Zhan Song. Applications of $k$-local MST for topology control and broadcasting in wireless ad hoc networks. IEEE Transactions on Parallel and Distributed Systems, 15(12):1057-1069, 2004.

[109] Nathan Linial. Locality in distributed graph algorithms. SIAM Journal on Computing, 21(1):193-201, 1992.

[110] László Lovász. Very large graphs, 2008. Manuscript, arXiv:0902.0132 [math.CO].

[111] Michael Luby. A simple parallel algorithm for the maximal independent set problem. SIAM Journal on Computing, 15(4):1036-1053, 1986.

[112] Michael Luby. Removing randomness in parallel computation without a processor penalty. Journal of Computer and System Sciences, 47(2):250-286, 1993.

[113] Michael Luby and Noam Nisan. A parallel approximation algorithm for positive linear programming. In Proc. 25th Annual ACM Symposium on Theory of Computing (STOC, San Diego, CA, USA, May 1993), pages 448-457. ACM Press, New York, NY, USA, 1993.

[114] Nancy A. Lynch. A hundred impossibility proofs for distributed computing. In Proc. 8th Annual ACM Symposium on Principles of Distributed Computing (PODC, Edmonton, Canada, August 1989), pages 1-28. ACM Press, New York, NY, USA, 1989.

[115] Alain Mayer, Moni Naor, and Larry Stockmeyer. Local computations on static and dynamic graphs. In Proc. 3rd Israel Symposium on the Theory of Computing and Systems (ISTCS, Tel Aviv, Israel, January 1995), pages 268-278. IEEE, Piscataway, NJ, USA, 1995.

[116] Michael Mitzenmacher and Eli Upfal. Probability and Computing: Randomized Algorithms and Probabilistic Analysis. Cambridge University Press, Cambridge, UK, 2005.

[117] Thomas Moscibroda. Locality, Scheduling, and Selfishness: Algorithmic Foundations of Highly Decentralized Networks. PhD thesis, ETH Zurich, 2006.

[118] Rajeev Motwani and Prabhakar Raghavan. Randomized Algorithms. Cambridge University Press, Cambridge, UK, 1995.

[119] James R. Munkres. Topology. Prentice Hall, Upper Saddle River, NJ, USA, 2nd edition, 2000.

[120] Moni Naor. A lower bound on probabilistic algorithms for distributive ring coloring. SIAM Journal on Discrete Mathematics, 4(3):409-412, 1991.

[121] Moni Naor and Larry Stockmeyer. What can be computed locally? SIAM Journal on Computing, 24(6):1259-1277, 1995.

[122] Huy N. Nguyen and Krzysztof Onak. Constant-time approximation algorithms via local improvements. In Proc. 49th Annual IEEE Symposium on Foundations of Computer Science (FOCS, Philadelphia, PA, USA, October 2008), pages 327-336. IEEE Computer Society Press, Los Alamitos, CA, USA, 2008.

[123] Christos H. Papadimitriou and Kenneth Steiglitz. Combinatorial Optimization: Algorithms and Complexity. Dover Publications, Inc., Mineola, NY, USA, 1998. 
[124] Christos H. Papadimitriou and Mihalis Yannakakis. On the value of information in distributed decision-making. In Proc. 10th Annual ACM Symposium on Principles of Distributed Computing (PODC, Montreal, Quebec, Canada, August 1991), pages 61-64. ACM Press, New York, NY, USA, 1991.

[125] Christos H. Papadimitriou and Mihalis Yannakakis. Linear programming without the matrix. In Proc. 25th Annual ACM Symposium on Theory of Computing (STOC, San Diego, CA, USA, May 1993), pages 121-129. ACM Press, New York, NY, USA, 1993.

[126] Michal Parnas and Dana Ron. Approximating the minimum vertex cover in sublinear time and a connection to distributed algorithms. Theoretical Computer Science, 381(1-3):183-196, 2007.

[127] David Peleg. Distributed Computing - A Locality-Sensitive Approach. Society for Industrial and Applied Mathematics, Philadelphia, PA, USA, 2000.

[128] Valentin Polishchuk and Jukka Suomela. A simple local 3-approximation algorithm for vertex cover. Information Processing Letters, 109(12):642-645, 2009.

[129] Frank P. Ramsey. On a problem of formal logic. Proceedings of the London Mathematical Society, 30:264-286, 1930.

[130] Johannes Schneider and Roger Wattenhofer. A log-star distributed maximal independent set algorithm for growth-bounded graphs. In Proc. 27th Annual ACM Symposium on Principles of Distributed Computing (PODC, Toronto, Canada, August 2008), pages 35-44. ACM Press, New York, NY, USA, 2008.

[131] Marco Schneider. Self-stabilization. ACM Computing Surveys, 25(1):45-67, 1993.

[132] Petra Šparl and Janez Žerovnik. 2-local 4/3-competitive algorithm for multicoloring hexagonal graphs. Journal of Algorithms, 55(1):29-41, 2005.

[133] Aaron D. Sterling. A limit to the power of multiple nucleation in self-assembly. In Proc. 22nd International Symposium on Distributed Computing (DISC, Arcachon, France, September 2008), volume 5218 of Lecture Notes in Computer Science, pages 451-465. Springer, Berlin, Germany, 2008.

[134] Jukka Suomela. Optimisation Problems in Wireless Sensor Networks: Local Algorithms and Local Graphs. PhD thesis, University of Helsinki, Department of Computer Science, Helsinki, Finland, May 2009.

[135] Jukka Suomela. Distributed algorithms for edge dominating sets. In Proc. 29th Annual ACM Symposium on Principles of Distributed Computing (PODC, Zurich, Switzerland, July 2010), pages 365-374. ACM Press, New York, NY, USA, 2010.

[136] Jukka Suomela. Survey of local algorithms. ACM Computing Surveys, 2011. To appear.

[137] Godfried T. Toussaint. The relative neighbourhood graph of a finite planar set. Pattern Recognition, 12(4):261-268, 1980.

[138] Jorge Urrutia. Local solutions for global problems in wireless networks. Journal of Discrete Algorithms, 5(3):395-407, 2007.

[139] Vijay V. Vazirani. Approximation Algorithms. Springer, Berlin, Germany, 2001.

[140] Yu Wang and Xiang-Yang Li. Localized construction of bounded degree and planar spanner for wireless ad hoc networks. Mobile Networks and Applications, 11(2):161$175,2006$.

[141] Mirjam Wattenhofer and Roger Wattenhofer. Distributed weighted matching. In Proc. 18th International Symposium on Distributed Computing (DISC, Amsterdam, Netherlands, October 2004), volume 3274 of Lecture Notes in Computer Science, pages 335-348. Springer, Berlin, Germany, 2004.

[142] Roger Wattenhofer and Aaron Zollinger. XTC: a practical topology control algorithm for ad-hoc networks. In Proc. 18th IEEE International Parallel and Distributed Processing Symposium (IPDPS, Santa Fe, NM, USA, April 2004). IEEE Computer Society Press, Los Alamitos, CA, USA, 2004.

[143] Paul M. Weichsel. The Kronecker product of graphs. Proceedings of the American Mathematical Society, 13(1):47-52, 1962.

[144] Andreas Wiese. Local approximation algorithms in unit disk graphs. Master's thesis, Technische Universität Berlin, 2007. 
[145] Andreas Wiese and Evangelos Kranakis. Impact of locality on location aware unit disk graphs. Algorithms, 1:2-29, 2008.

[146] Andreas Wiese and Evangelos Kranakis. Local maximal matching and local 2approximation for vertex cover in UDGs. In Proc. 7th International Conference on Ad-Hoc Networks 8 Wireless (AdHoc-NOW, Sophia Antipolis, France, September 2008), volume 5198 of Lecture Notes in Computer Science, pages 1-14. Springer, Berlin, Germany, 2008.

[147] Andreas Wiese and Evangelos Kranakis. Local construction and coloring of spanners of location aware unit disk graphs. Discrete Mathematics, Algorithms and Applications, 1(4):555-588, 2009.

[148] Andreas Wiese and Evangelos Kranakis. Local PTAS for dominating and connected dominating set in location aware unit disk graphs. In Proc. 6th Workshop on Approximation and Online Algorithms (WAOA, Karlsruhe, Germany, September 2008), volume 5426 of Lecture Notes in Computer Science, pages 227-240. Springer, Berlin, Germany, 2009.

[149] Andreas Wiese and Evangelos Kranakis. Local PTAS for independent set and vertex cover in location aware unit disk graphs. Ad Hoc 85 Sensor Wireless Networks: An International Journal, 7(3-4):273-293, 2009.

[150] Masafumi Yamashita and Tsunehiko Kameda. Computing on anonymous networks: Part I - characterizing the solvable cases. IEEE Transactions on Parallel and Distributed Systems, 7(1):69-89, 1996.

[151] Mihalis Yannakakis and Fanica Gavril. Edge dominating sets in graphs. SIAM Journal on Applied Mathematics, 38(3):364-372, 1980.

[152] Andrew Chi-Chih Yao. On constructing minimum spanning trees in $k$-dimensional spaces and related problems. SIAM Journal on Computing, 11(4):721-736, 1982.

[153] Aaron Zollinger. Geographic routing. In Ming-Yang Kao, editor, Encyclopedia of Algorithms. Springer, New York, NY, USA, 2008.

Jukka Suomela, Helsinki Institute for Information Technology HitT, University of Helsinki, P.O. Box 68, Fi-00014 University of Helsinki, Finland

E-mail address: jukka.suomela@cs.helsinki.fi 